\title{
THE RESTRICTION OPERATOR ON BERGMAN SPACES
}

\author{
DEBRAJ CHAKRABARTI AND SÖNMEZ ŞAHUTOĞLU
}

\begin{abstract}
We study the restriction operator from the Bergman space of a domain in $\mathbb{C}^{n}$ to the Bergman space of a non-empty open subset of the domain. We relate the restriction operator to the Toeplitz operator on the Bergman space of the domain whose symbol is the characteristic function of the subset. Using the biholomorphic invariance of the spectrum of the associated Toeplitz operator, we study the restriction operator from the Bergman space of the unit disc to the Bergman space of subdomains with large symmetry groups, such as horodiscs and subdomains bounded by hypercycles. Furthermore, we prove a sharp estimate of the norm of the restriction operator in case the domain and the subdomain are balls. We also study various operator theoretic properties of the restriction operator such as compactness and essential norm estimates.
\end{abstract}

\section{INTRODUCTION}

1.1. The restriction operator. Let $\Omega$ be a domain in $\mathbb{C}^{n}$ and $A^{2}(\Omega)$ be its Bergman space, the linear space of holomorphic functions which are square integrable with respect to the Lebesgue measure. Recall that $A^{2}(\Omega)$ is a closed subspace of $L^{2}(\Omega)$ and therefore is a Hilbert space with the induced inner product. If $U \subset \Omega$ is a nonempty open subset, one can define the restriction operator $R_{U}: A^{2}(\Omega) \rightarrow A^{2}(U)$ given by $R_{U} f=\left.f\right|_{U}$. Clearly, $R_{U}$ is a bounded linear map between Hilbert spaces. In some problems of complex analysis, one may want to know further properties of the operator $R_{U}$. For example, the non-compactness of $R_{U}$ in a special situation plays a crucial role in Fu and Straube's seminal study of the compactness of the $\bar{\partial}$-Neumann problem on convex domains (see [FS98]).

The goal of this paper is to study the operator $R_{U}$ by establishing a relation with the theory of Toeplitz operators on Bergman spaces. Using this relation, given any domain $\Omega$ in $\mathbb{C}^{n}$, and a non-empty open subset $U \subset \Omega$, we can define a spectrum of $U$ relative to $\Omega$ in such a way that this spectrum is an invariant of the complex geometry of $\Omega$. More precisely, if $\widetilde{\Omega}$ is a domain biholomorphic to $\Omega$, and $f: \Omega \rightarrow \widetilde{\Omega}$ is a biholomorphic mapping, then the spectrum of $U$ with respect to $\Omega$ is identical to the spectrum of $f(U)$ with respect to $\widetilde{\Omega}$. This clearly assumes a special interest when $f: \Omega \rightarrow \Omega$ is a biholomorphic automorphism of $\Omega$, and it follows that two subsets of $\Omega$ which are biholomorphically congruent (i.e., there is an automorphism of $\Omega$ mapping one to the other) are also isospectral. As we will see, the spectrum encodes important geometric and function theoretic information about the way the set $U$ "sits inside" $\Omega$.

The considerations of this paper extend easily to complex manifolds, provided we use the intrinsic Bergman spaces of square-integrable holomorphic $n$-forms. For ease of exposition we will confine ourselves to domains in $\mathbb{C}^{n}$. We will further assume that $A^{2}(\Omega)$ is non-trivial (that is, $A^{2}(\Omega) \neq\{0\}$ ), an assumption which is certainly satisfied when $\Omega$ is either bounded or biholomorphic to a bounded domain.

1.2. The associated Toeplitz operator. We introduce the operator

$$
T_{U}=R_{U}^{*} R_{U}: A^{2}(\Omega) \rightarrow A^{2}(\Omega),
$$

where $R_{U}^{*}: A^{2}(U) \rightarrow A^{2}(\Omega)$ is the Hilbert-space adjoint of $R_{U}$. The operator $T_{U}$ is clearly a positive selfadjoint operator on $A^{2}(\Omega)$, and it is not difficult to show that it is the Toeplitz operator on the Bergman

2010 Mathematics Subject Classification. Primary 32A36; Secondary 47B35.

Key words and phrases. Restriction operator, Toeplitz operator, Bergman space.

Debraj Chakrabarti was partially supported by grant from the National Science Foundation] (\#1600371), a collaboration grant from the Simons Foundation (\# 316632) and also by an Early Career internal grant from Central Michigan University. 
space $A^{2}(\Omega)$ with symbol the characteristic function $\chi_{U}$ of the open subset $U$. That is, for $f \in A^{2}(\Omega)$ :

$$
T_{U} f=P\left(\chi_{U} f\right),
$$

where $P: L^{2}(\Omega) \rightarrow A^{2}(\Omega)$ is the Bergman projection (see Proposition 1 below). It is natural to try to determine the spectrum of the selfadjoint operator $T_{U}$, which is precisely the spectrum of $U$ relative to $\Omega$ referred to above. As noted above the spectrum of $T_{U}$ is invariant under biholomorphisms of the ambient domain $\Omega$ (see Proposition 2 below).

1.3. Main results. Many properties of $R_{U}$ can be read off from the spectrum of $T_{U}$, and so a precise understanding of $T_{U}$ (and therefore $R_{U}$ ) involves of a description of the spectral decomposition of $T_{U}$. When this cannot be done, one might want to measure the "size" of $T_{U}$ relative to the identity operator on $A^{2}(\Omega)$. This gives us a way of measuring the "function-theoretic size" of $U$ relative to $\Omega$. Among such measures are the norm $\left\|T_{U}\right\|=\left\|R_{U}\right\|^{2}$, the essential norm $\left\|T_{U}\right\|_{e}$ and when $T_{U}$ is compact (i.e. $\left\|T_{U}\right\|_{e}=0$ ) the Schatten $p$-norms of $T_{U}$. Note that each of these measures is monotone in the set $U$, i.e., they increase with the set $U$.

It is also natural to consider for each open set $U \subset \Omega$ the complementary restriction $R_{\Omega \backslash \bar{U}}$ from $A^{2}(\Omega)$ to the open set $\Omega \backslash \bar{U}$. In order to avoid pathological situations, we will make the assumption that the boundary $\partial U$ of $U$ has zero Lebesgue measure. The following result relates some of these notions.

Theorem 1. Let $\Omega$ be a domain in $\mathbb{C}^{n}$ and $U$ be a non-empty open subset of $\Omega$ such that $A^{2}(\Omega)$ is infinite dimensional and the boundary of $U$ has zero Lebesgue measure. Then

(1) If $R_{U}$ is compact then $\left\|R_{U}\right\|<1$ and $\left\|R_{\Omega \backslash \bar{U}}\right\|_{e}=\left\|R_{\Omega \backslash \bar{U}}\right\|=1$.

(2) $\left\|R_{U}\right\|<1$ if and only if the complementary restriction $R_{\Omega \backslash \bar{U}}$ has closed range.

From part (1), it follows that if $\left\|R_{U}\right\|=1$, then $R_{U}$ (and therefore $T_{U}$ ) is non-compact. However, the converse is not true, i.e., there exist open sets $U \subset \Omega$ such that $\left\|R_{U}\right\|<1$ and $R_{U}$ is not compact. Examples for $n=1$ may be found in part (2) of Theorem 3 ("horocyclic strips") and also part (2) of Theorem 4 ("hypercyclic lunes"). In higher dimensions, it is even possible for $U$ to be a smoothly bounded subdomain of $\Omega$ touching $\Omega$ at a point. For instance, let $\Omega$ be a ball and $U \subset \Omega$ be a another ball with a common boundary point with $\Omega$. Then Theorem 2 below implies that $\left\|R_{U}\right\|<1$. However, $R_{U}$ is not compact (see [Str10, Lemma 4.27]).

Part (2) of Theorem 1 may be thought of as a generalized form of Hartogs phenomenon. The classical Hartogs phenomenon refers to the situation of a domain $\Omega$ and a subset $U \subset \Omega$, where each holomorphic function on $\Omega \backslash \bar{U}$ extends to a holomorphic function on $\Omega$. This happens, for instance, when $U$ is relatively compact in $\Omega$, and the complement $\Omega \backslash \bar{U}$ is connected (see, for instance [CS01, Theorem 3.1.2]). Hartogs phenomenon can happen only in dimensions $n \geq 2$. An $L^{2}$-version of Hartogs phenomenon consists of the situation when $R_{\Omega \backslash \bar{U}}$ is an isomorphism from $A^{2}(\Omega)$ to $A^{2}(\Omega \backslash \bar{U})$. In this case $R_{\Omega \backslash \bar{U}}$ has closed range equal to $A^{2}(\Omega)$, and therefore by part (2) of our result we have $\left\|R_{U}\right\|<1$. This suggests that restriction operators of norm less than one are more common in dimensions $\geq 2$. This is confirmed in Theorem 2 and Example 3 below.

Theorem 1 shows that it is important to determine when the norm of the restriction operator is strictly less than 1. In Section 5 we look at the problem of computing the norm when the boundaries of $\Omega$ and $U$ touch at a smooth point of both. Theorem 1 suggests that the results will be different for $n=1$ and $n \geq 2$. For $n=1$, in Proposition [10, we show that $\left\|R_{U}\right\|=1$, if $\partial \Omega$ and $\partial U$ touch at a $C^{1}$-smooth point. Note that if we consider a $U$ such that a non-smooth point of $\partial U$ touches $\partial \Omega$, then even in one dimension, we may have $\left\|R_{U}\right\|<1$ (see e.g. part (2) of Theorem 4 below).

We then consider the case when $U$ is a ball contained in the ball $\Omega$. For $n=1$ one can get an exact value of $\left\|R_{U}\right\|$ from Proposition 7 when $\bar{U} \subset \Omega$, where in fact we obtain the full spectral decomposition. When $n=1$ and $\partial U$ and $\partial \Omega$ touch at a point, the spectral decomposition of $T_{U}$ will be worked out in Theorem 3 below. The case $n \geq 2$ is considered in the following. 


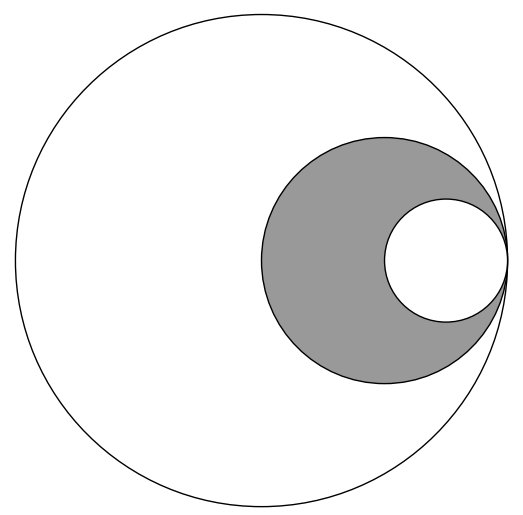

FiguRE 1. Horocyclic Strip

Theorem 2. Let $\Omega$ and $U$ be two balls in $\mathbb{C}^{n}$ of radii $R$ and $r$, respectively, such that $U \subset \Omega$, and let $\delta$ denote the distance from the center of $U$ to the boundary of $\Omega$. Then

$$
\left(\frac{r}{R}\right)^{n} \leq\left\|R_{U}\right\| \leq\left(\frac{\delta}{R}\right)^{\frac{n-1}{2}} .
$$

The inequalities are sharp in the sense that the lower bound is attained when $U$ and $\Omega$ are concentric and the upper bound is attained when the boundaries of the two balls touch at a point.

As is apparent from the examples in Section 3.2 below, when the ambient domain $\Omega$ and the subset $U$ have a large common symmetry group, the spectrum of $T_{U}$ can sometimes be determined explicitly. In this case, where we have Reinhardt symmetry, the operator $T_{U}$ has pure point spectrum. In Section 6 , we give a few other examples of subdomains of the unit disc for which there is sufficient symmetry to compute explicitly the spectrum of $T_{U}$. These computations are based on ideas of Vasilevski (see Vas08] and references therein) on spectral representation of algebras of Toeplitz operators. Below we describe these examples in detail, which apart from their intrinsic interest also provide simple counterexamples for conjectures related to the spectrum of $T_{U}$.

Let $\mathbb{D}$ be the unit disc in the plane, which we can think of as the hyperbolic plane with the Poincaré metric. Recall from hyperbolic geometry (see [Cox98, Chapter XI] for more information) that a horodisc in in the hyperbolic plane is represented in the Poincaré disc $\mathbb{D}$ by an Euclidean disc whose boundary is tangent to the unit circle. Intuitively, a horodisc is a "disc of infinite radius" in the hyperbolic plane. The boundary of a horodisc is called a horocycle, and the open set between two horocycles tangent to $\partial \mathbb{D}$ at the same point will be called a horocyclic strip. Therefore, for each $\alpha \in \mathbb{D}$, the set $\{z \in \mathbb{C}:|z-\alpha|<1-|\alpha|\}$ is a horodisc, and its boundary in $\mathbb{D},\{z \in \mathbb{C}:|z-\alpha|=1-|\alpha|,|z| \neq 1\}$, is a horocycle. We naturally call $\sqrt{1-|\alpha|}$ the Euclidean radius of the horocycle. The shaded region in Figure 1 shows the horocyclic strip

$$
\left\{z \in \mathbb{C}:\left|z-\frac{1}{2}\right|<\frac{1}{2},\left|z-\frac{3}{4}\right|>\frac{1}{4}\right\} .
$$

In the following theorem we characterize the spectrum of the restriction operator in case $U$ is a horodisc or a horocyclic strip in the unit disc.

Theorem 3. Let $\mathbb{D}$ be the unit disc in $\mathbb{C}$.

(1) If $U \subset \mathbb{D}$ is a horodisc, then the spectrum of $T_{U}$ is the full interval $[0,1]$.

(2) Let $0<\rho_{1}<\rho_{2}<1$ and $U \subset \mathbb{D}$ be a horocyclic strip between two horocycles of Euclidean radii $\rho_{1}$ and $\rho_{2}$ tangent to the unit circle at the same point. Then the spectrum of $T_{U}$ is the interval

$$
\left[0, \alpha^{-\frac{1}{\alpha-1}}-\alpha^{-\frac{\alpha}{\alpha-1}}\right]
$$




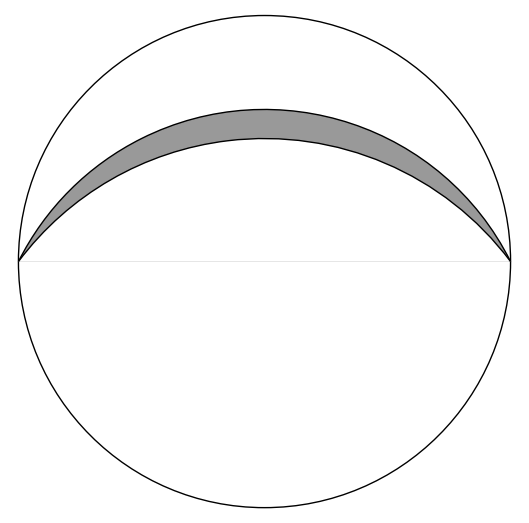

FiguRE 2. Region between two hypercycles

where

$$
\alpha=\frac{\frac{1}{\rho_{1}}-1}{\frac{1}{\rho_{2}}-1} .
$$

Consequently, the spectrum of $T_{U}$ consists only of essential points.

Another situation with $\Omega=\mathbb{D}$ in which one can compute the spectrum is the following. In hyperbolic plane geometry, given a hyperbolic geodesic $\Gamma$, a hypercycle or a equidistant-curve with axis $\Gamma$ is a curve consisting of points at a fixed signed distance from $\Gamma$ (see [Cox98, Chapter XI]). In the Poincaré disc model, the geodesic $\Gamma$ becomes a Euclidean circle or straight line which meets the bounding unit circle $\partial \mathbb{D}$ orthogonally, whereas a hypercycle becomes an arc of a Euclidean circle or a segment of a straight line which passes through the two points in $\bar{\Gamma} \cap \partial \mathbb{D}$. Hypercycles with the same axis may be referred to as coaxial. The region between two coaxial hypercycles will be referred to as a hypercyclic lune. The shaded region in Figure 2 shows the hypercyclic lune between two coaxial hypercycles with axis the diameter $(-1,1)$ of the unit circle, which is clearly a hyperbolic geodesic.

The ideal endpoints $\bar{\Gamma} \cap \partial \mathbb{D}$ of the geodesic $\Gamma$ divide the circle $\partial \mathbb{D}$ into two $\operatorname{arcs} C_{1}, C_{2}$. The region bounded by a hypercycle with axis $\Gamma$ and one of the $\operatorname{arcs} C_{j}$ will be called a hypercyclic crescent. The complement of the shaded hypercyclic lune of Figure 2 is the disjoint union of two hypercyclic crescents.

Theorem 4. Let $\mathbb{D}$ be the unit disc in $\mathbb{C}$.

(1) If $U \subset \mathbb{D}$ is a hypercyclic crescent, then the spectrum of $T_{U}$ is the full interval $[0,1]$.

(2) If $U \subset \mathbb{D}$ is a hypercyclic lune determined by two coaxial hypercycles, then the spectrum of $T_{U}$ is an interval of the form $\left[0,\left\|T_{U}\right\|\right]$ where $0<\left\|T_{U}\right\|<1$.

Observe that there is a one parameter group of biholomorphic automorphisms of the unit disc which maps a horocyclic strip (or a horodisc) to itself. Similarly, there is a one-parameter group of automorphisms of the unit disc which maps a hypercyclic lune to itself. Both assertions can be seen by mapping the unit disc onto the upper half plane. The presence of such a large group of automorphisms implies noncompactness of the restriction operator.

Theorem 5. Let $\Omega$ be a smooth bounded pseudoconvex domain in $\mathbb{C}^{n}$ and $U \subset \Omega$ be a non-empty open subset. Assume that there exists a closed noncompact subgroup $G$ of the topological group Aut $(\Omega)$ of biholomorphic automorphisms of $\Omega$ such that $f(U) \subset U$ for all $f \in G$. Then the operator $T_{U}$ is non-compact.

We note that the group of automorphisms $\operatorname{Aut}(\Omega)$ is naturally a topological group in the compact-open topology, and when $\Omega$ is bounded, this group has a natural Lie group structure. See [Nar95] for more details.

Theorem 5 is purely qualitative, and only gives the existence of an essential spectrum of $T_{U}$ without describing its nature. We recall that the essential spectrum is part of the spectrum that does not contain 
the eigenvalues with finite multiplicity (see, for example, [Dav95, pg 73]). In the absence of a large group of automorphisms of $\Omega$ which maps $U$ to itself, one cannot hope for a complete determination of the spectrum of $U$ relative to $\Omega$ along the lines of Theorems 3 or 4 . However, considering that $T_{U}$ is a Toeplitz operator on $A^{2}(\Omega)$, one can obtain further interesting information about the spectrum.

Theorems 3 and 4 may give the impression that regions in the disc which have cusps or corners on the boundary typically have noncompact restriction operators. A counterexample to this is shown in Figure 3. which shows an "ideal triangle" $\Delta$ in the Poincaré disc. This non-compact open set is bounded by three entire hyperbolic geodesics, and it is known from hyperbolic geometry that the hyperbolic area $A_{H}(\Delta)=\pi$, provided the metric is normalized to have Gaussian curvature -1 . In fact, in this case, not only are the operators $T_{U}$ and $R_{U}$ compact, but $T_{U}$ belongs to the trace class, i.e., the Schatten 1-norm of $T_{U}$

$$
\left\|T_{U}\right\|_{S_{1}}=\sum_{j=1}^{\infty} \lambda_{j}<\infty
$$

where $\left\{\lambda_{j}\right\}$ are the eigenvalues of the compact operator $T_{U}$. In fact, for a domain $\Omega \subset \mathbb{C}^{n}$ and an open set $U \subset \Omega$, the Schatten-1 norm of $T_{U}$ (i.e. trace of $T_{U}$ ) may be computed by the trace formula

$$
\left\|T_{U}\right\|_{S_{1}}=\int_{U} B_{\Omega}(\zeta, \zeta) d V(\zeta)
$$

where $B_{\Omega}$ is the Bergman kernel of $\Omega$ (see Section 8 for a proof). When $\Omega$ is homogeneous (for example the unit disc or ball) this is the same (up to a multiplicative constant) as the Riemannian volume of $U$ in the Bergman metric. Therefore, it follows that for homogenous domains $\Omega$, the volume of $U$ is a a spectral invariant. For the unit disc, we have the normalized volume element $d A_{H}(z)=\frac{4 d V(z)}{\left(1-|z|^{2}\right)^{2}}$. So it follows that the trace of the operator $T_{\Delta}$ on $A^{2}(\mathbb{D})$ is given by

$$
\left\|T_{\Delta}\right\|_{S_{1}}=\int_{\Delta} B(z, z) d V(z)=\int_{\Delta} \frac{1}{4 \pi} d A_{H}(z)=\frac{1}{4},
$$

so that the restriction operator $R_{U}$ belongs to the Schatten $\frac{1}{2}$-class, and

$$
\left\|R_{\Delta}\right\|_{S_{1 / 2}}=\sqrt{\left\|T_{\Delta}\right\|_{S_{1}}}=\frac{1}{2}
$$

In particular, we have the crude norm estimate

$$
\left\|R_{\Delta}\right\|<\frac{1}{4}
$$

More generally, we can measure the "degree of compactness" of the operators $R_{U}$ and $T_{U}$ using the Schatten p-norm $\left\|T_{U}\right\|_{S_{p}}$ and the fact that $\left\|R_{U}\right\|_{S_{p}}^{2}=\left\|T_{U}\right\|_{S_{2 p}}$ (see [Zhu07]). If $T_{U}$ is compact, since it is known to be positive and self-adjoint, we have

$$
\left\|T_{U}\right\|_{S_{p}}^{p}=\sum_{j=1}^{\infty} \lambda_{j}^{p},
$$

where $\left\{\lambda_{j}\right\}_{j=1}^{\infty}$ are the eigenvalues of $T_{U}$. For each $p$, the Schatten norm $\left\|T_{U}\right\|_{S_{p}}$ gives us a way of measuring the size of $U$ relative to $\Omega$. In view of (4) we can say that the Schatten norms represent "volumelike" invariants of the biholomorphic geometry of subdomains. In Section 8 , we consider the problem of computing Schatten norms of $R_{U}$ and $T_{U}$.

1.4. Historical note. As early as 1927, Stefan Bergman had developed the theory of "Doubly Orthogonal" sequences of functions in Bergman spaces, which implicitly involves the restriction operator (see [Ber70].) In [Sha79] this relationship was made precise (this is basically our Proposition [5).

When $U$ is a relatively compact subset of $\Omega$, compactness of $R_{U}$ follows immediately from Montel's Theorem. In [Gun66, page 61ff.] this was used in a proof of the finite dimensionality of cohomology groups of compact Riemann surfaces. Henkin and Mityagin in [MH71, Lemma 1.6] further showed that in this 


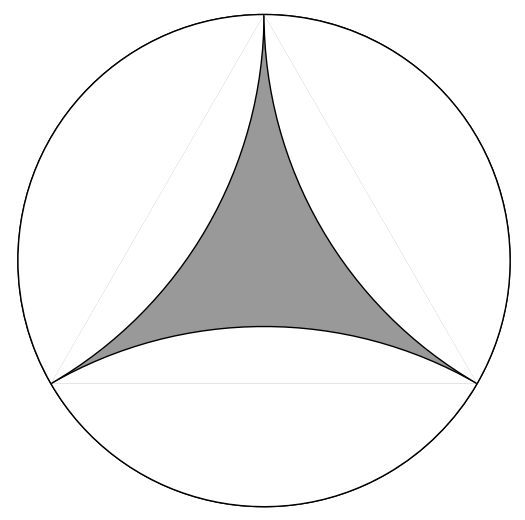

FiguRE 3. Ideal triangle

case the operator $R_{U}$ is in each Schatten class. In Lar86, the spectrum of the operator $T_{U}$ was studied when the ambient domain $\Omega$ is the unit disc and $U$ is a disjoint union of annuli, all centered at 0 . Related results were also studied in Lue81, Lue84.

The non-compactness of the restriction operator when $\Omega$ is a convex domain in $\mathbb{C}^{n}$ and $U$ is a dilated subdomain of $\Omega$ (with a common boundary point with $\Omega$ ) was used by Fu and Straube in [FS98] to study noncompactness of the $\bar{\partial}$-Neumann problem. These investigations were continued by Mijoung Kim in her thesis [Kim03] and in [Kim04, where $U$ is said to be "fat" or "thick" in $\Omega$ if $R_{U}$ is not compact. Further extensions of these ideas may be found in [SS06, Dal18].

In the work of Vasilevski (Vas08] and references therein), special cases of the operators $T_{U}$ on the Bergman space of the disc arise in a different context. When the set $U$ has certain symmetries, it follows that the operator $T_{U}$ belongs to a commutative subalgebra of Toeplitz operators. We use results from [Vas08 to compute the spectra of certain subsets of the unit disc below (see Section 6).

1.5. Acknowledgment. We thank Siqi Fu, Trieu Le, and Laszlo Lempert for helpful comments and discussions.

\section{BASIC FACTS}

2.1. Definitions and notation. For a domain $\Omega$ in $\mathbb{C}^{n}$, recall that we denote by $A^{2}(\Omega)$ the Bergman space of holomorphic functions $f$ on $\Omega$ such that

$$
\|f\|_{\Omega}^{2}=\int_{\Omega}|f|^{2} d V<\infty
$$

where $d V$ is the natural volume form of $\mathbb{C}^{n}$ (identified with $\mathbb{R}^{2 n}$ ). Then $A^{2}(\Omega)$ is a Hilbert space with the norm $\|\cdot\|_{\Omega}$.

Since $R_{U}: A^{2}(\Omega) \rightarrow A^{2}(U)$ is an injective bounded linear map between Hilbert spaces, the adjoint $R_{U}^{*}$ is a bounded linear map from $A^{2}(U)$ to $A^{2}(\Omega)$, which has dense range. Let $B_{\Omega}: \Omega \times \Omega \rightarrow \mathbb{C}$ denote the Bergman kernel of $\Omega$. Recall that the operator $T_{U}$ on $A^{2}(\Omega)$ is defined to be the composition $R_{U}^{*} R_{U}$.

Proposition 1. Let $\Omega$ be a domain in $\mathbb{C}^{n}$ and $U$ be a non-empty open subset of $\Omega$. Then for $g \in A^{2}(\Omega)$, we have

$$
T_{U} g=P\left(\chi_{U} g\right)
$$

where $P: L^{2}(\Omega) \rightarrow A^{2}(\Omega)$ is the Bergman projection and $\chi_{U}$ is the characteristic function of the set $U$. Furthermore, the operator $T_{U}$ is injective and its range is dense in $A^{2}(\Omega)$. 
Proof. Let $f \in A^{2}(U)$. We will first show that the adjoint operator $R_{U}^{*}: A^{2}(U) \rightarrow A^{2}(\Omega)$ is given by the formula

$$
R_{U}^{*} f(z)=\int_{U} f(w) B_{\Omega}(z, w) d V(w) .
$$

Notice that this may be written in the form $R_{U}^{*} f=P(\widetilde{f})$, where $\widetilde{f} \in L^{2}(\Omega)$ is the function which coincides with $f$ on $U$ and is zero on $\Omega \backslash U$. This shows that the right hand side of (5) defines a bounded linear map from $A^{2}(U)$ to $A^{2}(\Omega)$. To prove (15), let $g \in A^{2}(\Omega)$. Then

$$
\left\langle R_{U}^{*} f, g\right\rangle_{A^{2}(\Omega)}=\left\langle f, R_{U} g\right\rangle_{A^{2}(U)}=\langle\tilde{f}, g\rangle_{L^{2}(\Omega)}=\langle P(\tilde{f}), g\rangle_{A^{2}(\Omega)} .
$$

We note that the last equality follows on decomposing $\widetilde{f}$ into its components along $A^{2}(\Omega)$ and $A^{2}(\Omega)^{\perp}$. This shows that $R_{U}^{*} f-P(\widetilde{f}) \in A^{2}(\Omega)$ is orthogonal to each element of $A^{2}(\Omega)$. Hence, $R_{U}^{*} f-P(\widetilde{f})=0$ which establishes (5).

For $g \in A^{2}(\Omega)$ and $z \in \Omega$ we now have

$$
\begin{aligned}
T_{U} g(z) & =R_{U}^{*}\left(R_{U} g\right)(z) \\
& =\int_{U} g(w) B_{\Omega}(z, w) d V(w) \\
& =\int_{\Omega}\left(\chi_{U}(w) g(w)\right) B_{\Omega}(z, w) d V(w) \\
& =P\left(\chi_{U} g\right)(z)
\end{aligned}
$$

Finally we prove that $T_{U}$ has dense range. Connectedness of $\Omega$ together with the identity principle imply that the operator $R_{U}$ is injective. Then we conclude that $T_{U}$ is injective because if $T_{U} f=0$ for some $f \in A^{2}(\Omega)$, then $\left\langle T_{U} f, f\right\rangle_{\Omega}=\left\|R_{U} f\right\|^{2}=0$ which, in turn, implies that $f=0$. Finally, since $T_{U}$ is injective, it follows that its adjoint $T_{U}=\left(T_{U}\right)^{*}$ has dense range.

Proposition 1 shows that $T_{U}$ is the Toeplitz operator on the Bergman space $A^{2}(\Omega)$ with symbol $\chi_{U}$ (see [Zhu07]). It is clear that $T_{U}$ is strictly positive. Note that the operators $R_{U}, R_{U}^{*}, T_{U}$ are simultaneously compact or non-compact (cf. [Zhu07, Theorems 1.16, 1.17]). Similarly, the operators $R_{U}, R_{U}^{*}, T_{U}$ simultaneously have closed range or do not have closed range. Furthermore,

$$
\left\|R_{U}\right\|=\left\|R_{U}^{*}\right\|=\sqrt{\left\|T_{U}\right\|}
$$

2.2. Biholomorphic invariance. Let $\phi: \widetilde{\Omega} \rightarrow \Omega$ be a biholomorphic map. We can associate an isometric isomorphism of Bergman spaces

$$
\phi^{\sharp}: A^{2}(\Omega) \rightarrow A^{2}(\widetilde{\Omega})
$$

by defining for $f \in A^{2}(\Omega)$ and $w \in \widetilde{\Omega}$

$$
\left(\phi^{\sharp} f\right)(w)=\operatorname{det} \phi^{\prime}(w) \cdot f(\phi(w))
$$

where $\phi^{\prime}(w): \mathbb{C}^{n} \rightarrow \mathbb{C}^{n}$ is the $\mathbb{C}$-linear map which is the complex derivative of $\phi$ at $w$. It follows from the change of variables formula that $\phi^{\sharp}$ is an isometric isomorphism of Hilbert spaces. Consequently, we have

$$
\left(\phi^{\sharp}\right)^{*} \cdot \phi^{\sharp}=\operatorname{id}_{A^{2}(\Omega)} \cdot
$$

Proposition 2. Let $\phi: \widetilde{\Omega} \rightarrow \Omega$ be a biholomorphic map, and let $\widetilde{U}$ is an open subset of $\widetilde{\Omega}$ such that $\phi(\widetilde{U})=U$. Then we have

$$
T_{\widetilde{U}}=\phi^{\sharp} \circ T_{U} \circ\left(\phi^{\sharp}\right)^{-1} .
$$

In particular, the self-adjoint operators $T_{U}$ and $T_{\widetilde{U}}$ are isospectral. 
Proof. It is clear that the following diagram commutes.

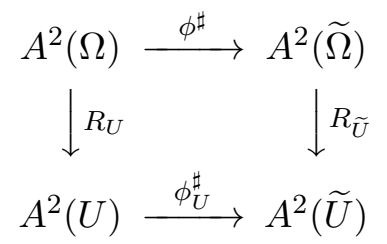

where $\left(\phi_{U}^{\sharp} f\right)(w)=\operatorname{det} \phi^{\prime}(w) \cdot f(\phi(w))$ for $f \in A^{2}(U)$ and $w \in \widetilde{U}$. That is, $\phi_{U}^{\sharp}=\left(\left.\phi\right|_{U}\right)^{\sharp}$. It follows that $R_{\widetilde{U}}=\phi_{U}^{\sharp} \circ R_{U} \circ\left(\phi^{\sharp}\right)^{-1}$. Then

$$
\begin{aligned}
T_{\widetilde{U}} & =\left(\phi_{U}^{\sharp} \circ R_{U} \circ\left(\phi^{\sharp}\right)^{-1}\right)^{*} \circ\left(\phi_{U}^{\sharp} \circ R_{U} \circ\left(\phi^{\sharp}\right)^{-1}\right) \\
& =\left(\left(\phi^{\sharp}\right)^{-1}\right)^{*} \circ R_{U}^{*} \circ\left(\phi_{U}^{\sharp}\right)^{*} \circ \phi_{U}^{\sharp} \circ R_{U} \circ\left(\phi^{\sharp}\right)^{-1} \\
& =\left(\left(\phi^{\sharp}\right)^{-1}\right)^{*} \circ R_{U}^{*} \circ R_{U} \circ\left(\phi^{\sharp}\right)^{-1} \\
& =\phi^{\sharp} \circ T_{U} \circ\left(\phi^{\sharp}\right)^{-1} .
\end{aligned}
$$

Hence, the proof of the proposition is complete.

Note that for any measurable subset $U \subset \Omega$, the formula

$$
T_{U}=P \circ m_{\chi_{U}}
$$

(where $P: L^{2}(\Omega) \rightarrow A^{2}(\Omega)$ is the Bergman projection and $m_{\chi_{U}}: A^{2}(\Omega) \rightarrow L^{2}(\Omega)$ is the multiplication operator induced by $\chi_{U}$ ) defines a positive-definite self-adjoint Toeplitz operator satisfying the invariance property of Proposition 2. Therefore, this allows us to extend the notion of spectrum of a subset from open subsets of $\Omega$ to arbitrary measurable subsets of $\Omega$. However, since we are motivated by the relation of $T_{U}$ with the restriction operator $R_{U}$, we will continue to assume that $U$ is open.

2.3. Intrinsic Bergman spaces. This invariance property is a manifestation of the fact that it is possible to define intrinsic versions of the Bergman space and the operators $R_{U}, R_{U}^{*}, T_{U}$ without any reference to a given volume form $d V$.

Let $\Omega$ be a complex manifold of dimension $n$. We denote the intrinsic Bergman space of $\Omega$ by $A_{n, 0}^{2}(\Omega)$. This is, by definition the Hilbert space, of those holomorphic $(n, 0)$-forms $f$ on $\Omega$ such that

$$
\|f\|_{\Omega}^{2}=(\sqrt{-1})^{n^{2}} \int_{\Omega} f \wedge \bar{f}<\infty
$$

If $\Omega$ is a domain in $\mathbb{C}^{n}$, the Hilbert space is clearly $A_{n, 0}^{2}(\Omega)$ is isometrically isomorphic to $A^{2}(\Omega)$ in a natural way. If $\iota_{U}: U \rightarrow \Omega$ is the inclusion map, the pullback operator $\iota_{U}^{*}: A_{n, 0}^{2}(\Omega) \rightarrow A_{n, 0}^{2}(U)$ is defined invariantly, i.e. without any reference to a distinguished metric or volume form on $\Omega$. The identification of $A^{2}(\Omega)$ with $A_{n, 0}^{2}(\Omega)$ given above identifies the operator $R_{U}$ with the invariantly defined operator $\iota_{U}^{*}$. Similarly, one can give invariant descriptions of $R_{U}^{*}, S_{U}$ and $T_{U}$. Note that these invariant descriptions immediately show that the analytic properties of these operators are invariant under biholomorphisms, as seen in Proposition 2 ,

\section{Compact REStriction Operators}

3.1. Eigenvalues and eigenvectors. In this section we explore some spectral properties of $T_{U}$ when there are eigenvalues in the spectrum.

Proposition 3. Let $\Omega$ be a domain in $\mathbb{C}^{n}$ with nontrivial Bergman space and $U$ be a non-empty open subset of $\Omega$ such that $\Omega \backslash \bar{U} \neq \emptyset$. Suppose that $\lambda$ is an eigenvalue of $T_{U}$. Then

$$
0<\lambda<1 \text {. }
$$

Furthermore, whenever 0 or 1 is in the spectrum of $T_{U}$, it belongs to the essential spectrum of $T_{U}$. 
Proof. Let $\lambda$ be the eigenvalue of $T_{U}$ and $\phi$ is a corresponding eigenfunction. Then

$$
\langle\phi, \phi\rangle_{U}=\left\langle R_{U} \phi, R_{U} \phi\right\rangle_{U}=\left\langle R_{U}^{*} R_{U} \phi, \phi\right\rangle_{\Omega}=\left\langle T_{U} \phi, \phi\right\rangle=\lambda\langle\phi, \phi\rangle_{\Omega}
$$

Then $\lambda=\frac{\|\phi\|_{U}^{2}}{\|\phi\|_{\Omega}^{2}}$. Using the facts that $\Omega \backslash \bar{U} \neq \emptyset$, and that $\phi$ is a nonzero holomorphic function, we see that

$$
0<\langle\phi, \phi\rangle_{U}<\langle\phi, \phi\rangle_{\Omega}
$$

It follows that $0<\lambda<1$. So, if 0 or 1 is in the spectrum of $T_{U}$ then it is not an eigenvalue and, hence, it belongs to the essential spectrum of $T_{U}$.

We now suppose that $T_{U}$ has pure point spectrum, i.e., there is an orthonormal basis of $A^{2}(\Omega)$ consisting of eigenvectors of $T_{U}$. If $\left\{\phi_{j}\right\}$ is an orthonormal basis of $A^{2}(\Omega)$ consisting of eigenvectors of $T_{U}$ and $\lambda_{j}$ is the eigenvalue corresponding to $\phi_{j}$, then it is clear that

$$
\operatorname{Spec} T_{U}=\overline{\left\{\lambda_{j}\right\}_{j=1}^{\infty}}
$$

the closure of the set of eigenvalues. Furthermore, for each $f \in A^{2}(\Omega)$ we can write,

$$
T_{U} f=\sum_{j=0}^{\infty} \lambda_{j}\left\langle f, \phi_{j}\right\rangle \phi_{j},
$$

where the series converges in the norm of $A^{2}(\Omega)$.

In order not to repeat many times the conclusion (1) of Proposition 5, let us say that an orthonormal basis $\left\{\phi_{j}\right\}$ of $A^{2}(\Omega)$ is adapted to an open subset $U \subset \Omega$, if $\left\{\left.\phi_{j}\right|_{U}\right\}$ is an orthogonal set in $A^{2}(U)$, i.e., if $j \neq k$, then

$$
\left\langle\phi_{j}, \phi_{k}\right\rangle_{U}=\int_{U} \phi_{j}(z) \overline{\phi_{k}(z)} d V(z)=0 .
$$

In the literature, one usually expresses this by saying that the family $\left\{\phi_{j}\right\}$ forms a doubly orthogonal sequence of functions (with respect to the two domains $\Omega$ and $U$ ). Doubly orthogonal systems were discovered and named by Stefan Bergman as far back as 1927 (see [Ber70]). For some recent results related to doubly orthogonal systems see [And00, AAD01, GPS03, PP06].

Proposition 4. Let $\Omega$ be a domain in $\mathbb{C}^{n}$ with nontrivial Bergman space, $U$ be a non-empty open subset of $\Omega$, and $\left\{\phi_{j}\right\}$ be an orthonormal basis of $A^{2}(\Omega)$. Then the following are equivalent.

(1) $\left\{\phi_{j}\right\}$ is adapted to $U$,

(2) $T_{U}$ has pure point spectrum and each $\phi_{j}$ is an eigenvector of $T_{U}$.

If either condition (1) or (2) (and therefore both) are satisfied, the eigenvalue $\lambda_{j}$ of $T_{U}$ corresponding to the eigenvector $\phi_{j}$ is given by

$$
\lambda_{j}=\left\langle\phi_{j}, \phi_{j}\right\rangle_{U}
$$

Proof. First we will prove that (1) implies (2). Assume that $\left\{\phi_{j}\right\}$ is adapted to $U$. Then for any $j, k$ we have

$$
\left\langle T_{U} \phi_{j}, \phi_{k}\right\rangle_{\Omega}=\left\langle R_{U}^{*} R_{U} \phi_{j}, \phi_{k}\right\rangle_{\Omega}=\left\langle R_{U} \phi_{j}, R_{U} \phi_{k}\right\rangle_{\Omega}=\left\langle\phi_{j}, \phi_{k}\right\rangle_{U}=\left\langle\phi_{j}, \phi_{j}\right\rangle_{U} \delta_{j k}
$$

Since $\left\{\phi_{j}\right\}$ is an orthonormal basis, this means that $T_{U} \phi_{j}=\left\langle\phi_{j}, \phi_{j}\right\rangle_{U} \phi_{j}$ for each $j$. It follows that $\phi_{j}$ is an eigenvector of $T_{U}$ with eigenvalue $\lambda_{j}=\left\langle\phi_{j}, \phi_{j}\right\rangle_{U}$. Since $\left\{\phi_{j}\right\}$ is an orthonormal basis of $A^{2}(\Omega)$ it follows that $T_{U}$ has pure point spectrum.

To prove the converse, let us assume (2), and so $T_{U} \phi_{j}=\lambda_{j} \phi_{j}$, where $\lambda_{j}$ is the eigenvalue of $T_{U}$ corresponding to $\phi_{j}$. Then we have

$$
\left\langle\phi_{j}, \phi_{k}\right\rangle_{U}=\left\langle R_{U} \phi_{j}, R_{U} \phi_{k}\right\rangle_{U}=\left\langle R_{U}^{*} R_{U} \phi_{j}, \phi_{k}\right\rangle_{\Omega}=\left\langle T_{U} \phi_{j}, \phi_{k}\right\rangle=\lambda_{j}\left\langle\phi_{j}, \phi_{k}\right\rangle_{\Omega}=\lambda_{j} \delta_{j k}
$$

for all $j$ and $k$. Therefore, the family $\left\{\left.\phi_{j}\right|_{U}\right\}$ is orthogonal in $A^{2}(U)$, and $\lambda_{j}=\left\langle\phi_{j}, \phi_{j}\right\rangle_{U}$. 
The next proposition, first proved by Bergman (see [Ber70]) was proved by Shapiro ([Sha79]) by an application of the spectral theorem for compact self-adjoint operators, and describes the consequences of compactness of $R_{U}$ (or equivalently, that of $T_{U}=R_{U}^{*} R_{U}$ ).

Proposition 5 (Bergman-Shapiro). Let $\Omega$ be a domain in $\mathbb{C}^{n}$ with nontrivial Bergman space and $U$ be a non-empty open subset of $\Omega$. Suppose that the restriction operator $R_{U}: A^{2}(\Omega) \rightarrow A^{2}(U)$ is compact. Then

(1) there is an orthonormal basis $\left\{\phi_{j}\right\}$ of $A^{2}(\Omega)$ such that the restrictions $R_{U}\left(\phi_{j}\right)=\left.\phi_{j}\right|_{U}$ form an orthogonal set in $A^{2}(U)$;

(2) the operator $T_{U}$ has the following spectral representation as a series converging in $A^{2}(\Omega)$.

$$
T_{U} f=\sum_{j=1}^{\infty} \lambda_{j}\left\langle f, \phi_{j}\right\rangle_{\Omega} \phi_{j}, \quad\left(f \in A^{2}(\Omega)\right)
$$

where the eigenvalues are given by

$$
\lambda_{j}=\left\langle\phi_{j}, \phi_{j}\right\rangle_{U}=\int_{U}\left|\phi_{j}\right|^{2} d V
$$

Here, $\langle\cdot, \cdot\rangle_{\Omega}$ (resp. $\langle\cdot, \cdot\rangle_{U}$ ) denoted the inner product of $A^{2}(\Omega)$ (resp. $A^{2}(U)$.) We apply Proposition 5 to compute some spectra in highly symmetric situations in Examples 1 and 2, and in Proposition 7 ,

We provide a proof of Proposition 5 here for the convenience of the reader.

Proof of Proposition 5. First we will prove (1). Since $R_{U}$ is compact, then $T_{U}=R_{U}^{*} R_{U}$ is a compact self-adjoint operator, and by the spectral theorem, there is an orthonormal basis $\left\{\phi_{j}\right\}$ of $A^{2}(\Omega)$ consisting of eigenvectors of $T_{U}$. The implication $(2) \Rightarrow(1)$ of Proposition 4 immediately gives us the result.

Assertion (2) now follows on noting that in the spectral representation (9), when the orthonormal basis $\left\{\phi_{j}\right\}$ is adapted to $U$, the eigenvalues are given by (10).

\subsection{Some examples.}

Example 1 . Let $\mathbb{Z}_{+}=\{0,1,2,3, \ldots\}$ and suppose that $\Omega$ is a complete Reinhardt domain in $\mathbb{C}^{n}$ and $U \subset \Omega$ is a Reinhardt subdomain. Then for each multi-index $\alpha \in \mathbb{Z}_{+}^{n}$, we consider the function $\phi_{\alpha} \in A^{2}(\Omega)$ given by

$$
\phi_{\alpha}(z)=\frac{z^{\alpha}}{\left\|z^{\alpha}\right\|_{\Omega}} .
$$

Then $\left\{\phi_{\alpha}\right\}$ is an orthonormal basis of $A^{2}(\Omega)$. Further, since $U$ is also Reinhardt, it follows that $\left\langle\phi_{\alpha}, \phi_{\beta}\right\rangle_{U}=$ 0 if $\alpha \neq \beta$. Hence the orthonormal basis $\left\{\phi_{\alpha}\right\}$ is adapted to $U$ and the eigenvalues of $T_{U}$ are given by the ratios

Therefore, we have

$$
\lambda_{\alpha}=\frac{\left\|z^{\alpha}\right\|_{U}^{2}}{\left\|z^{\alpha}\right\|_{\Omega}^{2}} .
$$

$$
\left\|R_{U}\right\|=\sqrt{\left\|T_{U}\right\|}=\sup _{\alpha \in \mathbb{Z}_{+}^{n}} \frac{\left\|z^{\alpha}\right\|_{U}}{\left\|z^{\alpha}\right\|_{\Omega}} .
$$

Example 2. Let us now specialize to the case when $U$ is a dilated version of the complete Reinhardt domain $\Omega$, i.e., there is a $0<\rho<1$ such that $U=\rho \Omega=\{\rho z \mid z \in \Omega\}$. Then for each multi-index $\alpha \in \mathbb{Z}_{+}^{n}$, we have

$$
\begin{aligned}
\left\|z^{\alpha}\right\|_{U}^{2} & =\int_{U}\left|w^{\alpha}\right|^{2} d V(w) \\
& =\int_{\Omega}\left|(\rho z)^{\alpha}\right|^{2} \rho^{2 n} d V(z) \\
& =\rho^{2|\alpha|+2 n} \int_{\Omega}\left|z^{\alpha}\right|^{2} d V(z) \\
& =\rho^{2(|\alpha|+n)}\left\|z^{\alpha}\right\|_{\Omega}^{2} .
\end{aligned}
$$


Consequently, the eigenvalues of $T_{U}$ are

$$
\lambda_{\alpha}=\rho^{2(|\alpha|+n)}, \alpha \in \mathbb{Z}_{+}^{n} .
$$

Therefore, the norm of the restriction operator is given by

$$
\left\|T_{U}\right\|=\left\|R_{U}\right\|^{2}=\sup _{\alpha} \lambda_{\alpha}=\rho^{2 n} .
$$

We can now recapture a classic fact about annuli.

Proposition 6. For $0<r_{2}<r_{1}$, let $A\left(r_{1}, r_{2}\right)=\left\{z \in \mathbb{C}: r_{2}<|z|<r_{1}\right\}$ be an annulus in the plane. If $A\left(r_{1}^{\prime}, r_{2}^{\prime}\right)$ is conformally equivalent to $A\left(r_{1}, r_{2}\right)$, then

$$
\frac{r_{2}}{r_{1}}=\frac{r_{2}^{\prime}}{r_{1}^{\prime}}
$$

Proof. Let $f: A\left(r_{1}, r_{2}\right) \rightarrow A\left(r_{1}^{\prime}, r_{2}^{\prime}\right)$ be a conformal map, and let us set $\Omega=\left\{z \in \mathbb{C}:|z|<r_{1}\right\}, U=\{z \in$ $\left.\mathbb{C}:|z|<r_{2}\right\}, \widetilde{\Omega}=\left\{z \in \mathbb{C}:|z|<r_{1}^{\prime}\right\}, \widetilde{U}=\left\{z \in \mathbb{C}:|z|<r_{2}^{\prime}\right\}$. Notice that $U=\left(\frac{r_{2}}{r_{1}}\right) \Omega$ and $\widetilde{U}=\left(\frac{r_{2}^{\prime}}{r_{1}^{\prime}}\right) \widetilde{\Omega}$, so that thanks to (15), we have $\left\|T_{U}\right\|=\left(\frac{r_{2}}{r_{1}}\right)^{2}$ and $\left\|T_{\widetilde{U}}\right\|=\left(\frac{r_{2}^{\prime}}{r_{1}^{\prime}}\right)^{2}$.

Applying an inversion if necessary, we can assume that as $|f(z)| \rightarrow r_{1}^{\prime}$ as $|z| \rightarrow r_{1}$. We can now apply repeated Schwarz reflection in the inner circles, followed by an appeal to the Riemann removable singularity theorem to extend the map $f$ to a biholomorphic map from $\Omega$ to $\widetilde{\Omega}$ such that $f(U)=\widetilde{U}$. It follows that $\left\|T_{U}\right\|=\left\|T_{\widetilde{U}}\right\|$, and therefore $r_{2} / r_{1}=r_{2}^{\prime} / r_{1}^{\prime}$.

We now compute the spectrum of $T_{U}$ for an arbitrary relatively compact subdisc of the unit disc $\mathbb{D}$.

Proposition 7. Let $z_{0} \in \mathbb{C}, r>0, A=\sqrt{\frac{\left(1+\left|z_{0}\right|+r\right)\left(1-\left|z_{0}\right|+r\right)}{\left(1-\left|z_{0}\right|-r\right)\left(1+\left|z_{0}\right|-r\right)}}$, and $U=\left\{z \in \mathbb{C}:\left|z-z_{0}\right|<r\right\}$ such that $\bar{U} \Subset \Omega=\mathbb{D}$. Then the spectrum of $T_{U}$ is composed of eigenvalues $\left\{\lambda_{k}\right\}_{k=0}^{\infty}$ where

$$
\lambda_{k}=\left(\frac{A-1}{A+1}\right)^{2 k+2} .
$$

Proof. Recall that the distance in the Poincaré hyperbolic metric of the disc (suitably normalized) from the origin to the point $z \in \mathbb{D}$ is given by

$$
\eta(0, z)=\log \frac{1+|z|}{1-|z|}
$$

It follows that the disc $\{z \in \mathbb{C}:|z|<r\}$ is a hyperbolic disc centered at the origin and of radius $\eta(0, r)$. Since any two hyperbolic discs of the same radius are congruent under Aut $(\mathbb{D})$, it follows from (14) that the spectrum of a hyperbolic disk of radius $\rho$ consists of the points $\left\{r^{2 k+2}, k=0,1, \ldots\right\}$, where $r>0$ is such that $\rho=\eta(0, r)$. A computation using the formula for $\eta$ shows that

$$
r=\frac{e^{\rho}-1}{e^{\rho}+1}
$$

Now consider the disc $U=\left\{z \in \mathbb{C}:\left|z-z_{0}\right|<r\right\}$. Let $P$ and $Q$ be the points at which the diameter through $z_{0}$ meets the circumference of $U$, so that $|P|=\left|z_{0}\right|+r$ and $|Q|=|| z_{0}|-r|$ (if $z_{0}=0$, take any diameter). If $\left|z_{0}\right| \geq r$, then $0 \notin U$, and the hyperbolic radius of $U$ (thought of as a hyperbolic disc) is given by

$$
\begin{aligned}
\frac{1}{2}(\eta(0, P)-\eta(0, Q)) & =\frac{1}{2} \log \left(\frac{1+|| z_{0}|+r|}{1-|| z_{0}|+r|} \cdot \frac{1-|| z_{0}|-r|}{1+|| z_{0}|-r|}\right) \\
& =\frac{1}{2} \log \left(\frac{1+\left|z_{0}\right|+r}{1-\left|z_{0}\right|-r} \cdot \frac{1-\left|z_{0}\right|+r}{1+\left|z_{0}\right|-r}\right) \\
& =\log A
\end{aligned}
$$


Now, if $\left|z_{0}\right|<r$, then $0 \in U$, and in this case, the hyperbolic radius of $U$ is given by

$$
\begin{aligned}
\frac{1}{2}(\eta(0, P)+\eta(0, Q)) & =\frac{1}{2} \log \left(\frac{1+|| z_{0}|+r|}{1-|| z_{0}|+r|} \cdot \frac{1+|| z_{0}|-r|}{1-|| z_{0}|-r|}\right) \\
& =\frac{1}{2} \log \left(\frac{1+\left|z_{0}\right|+r}{1-\left|z_{0}\right|-r} \cdot \frac{1-\left|z_{0}\right|+r}{1+\left|z_{0}\right|-r}\right) \\
& =\log A .
\end{aligned}
$$

Hence the proof of the proposition is complete.

\section{Complementary domains and proof of Theorem 1}

\subsection{Spectral properties of complementary domains.}

Proposition 8. Let $\Omega$ be a domain in $\mathbb{C}^{n}$ with nontrivial Bergman space and $U$ be a non-empty open subset of $\Omega$ such that $\Omega \backslash \bar{U} \neq \emptyset$. Assume that $\partial U$ has zero Lebesgue measure. Then

(1) $T_{U}$ and $T_{\Omega \backslash \bar{U}}$ commute as operators on $A^{2}(\Omega)$ and

$$
\operatorname{Spec}\left(T_{\Omega \backslash \bar{U}}\right)=\left\{1-\lambda \mid \lambda \in \operatorname{Spec}\left(T_{U}\right)\right\},
$$

(2) if there is an orthonormal basis $\left\{\phi_{j}\right\}$ of $A^{2}(\Omega)$ adapted to $U$, this basis is also adapted to $\Omega \backslash \bar{U}$.

Proof. By Proposition 1 we see that for each $f \in A^{2}(\Omega)$ we have since $\chi_{U}+\chi_{\Omega \backslash \bar{U}}=1$ a.e.

$$
\begin{aligned}
T_{U} f(z)+T_{\Omega \backslash \bar{U}} f(z) & =P\left(\chi_{U} f\right)+P\left(\chi_{\Omega \backslash \bar{U}} f\right) \\
& =P\left(\left(\chi_{U}+\chi_{\Omega \backslash \bar{U}}\right) f\right) \\
& =P(f) \\
& =f .
\end{aligned}
$$

Then we have

$$
T_{\Omega \backslash \bar{U}}=\mathrm{id}_{A^{2}(\Omega)}-T_{U} .
$$

It follows that $T_{U}$ and $T_{\Omega \backslash \bar{U}}$ commute. Consequently $\operatorname{Spec}\left(T_{\Omega \backslash \bar{U}}\right)=\operatorname{Spec}\left(I-T_{U}\right)$ and (16) follows.

Assuming that $\left\{\phi_{j}\right\}$ is adapted to $U$, the fact that it is adapted to $\Omega \backslash \bar{U}$ follows on writing

$$
\left\langle\phi_{j}, \phi_{k}\right\rangle_{\Omega}=\left\langle\phi_{j}, \phi_{k}\right\rangle_{U}+\left\langle\phi_{j}, \phi_{k}\right\rangle_{\Omega \backslash \bar{U}}
$$

Hence the proof of the proposition is complete.

Next we prove Theorem 1 .

Proof of Theorem 1, First consider part (1). Assume that $R_{U}$ is compact. We observe that since $\left\|R_{U}\right\|=$ $\sqrt{\left\|T_{U}\right\|}$ and $\left\|T_{U}\right\|$ is the largest eigenvalue of $T_{U}$, which is strictly less than 1 by (8). Hence, we conclude that $\left\|R_{U}\right\|<1$.

To prove that $\left\|R_{\Omega \backslash \bar{U}}\right\|_{e}=1$, note that the operator $T_{U}$ is compact since $R_{U}$ is compact. Then 0 is an essential point of the spectrum of $T_{U}$, by Proposition 3 , Let $\left\{\phi_{j}\right\}$ be an orthonormal basis of $A^{2}(\Omega)$ consisting of eigenvectors of $T_{U}$, and let $\lambda_{j}$ be the eigenvalue of $T_{U}$ corresponding to the eigenvector $\phi_{j}$. It now follows from Proposition 8 that the operator $T_{\Omega \backslash \bar{U}}$ has pure point spectrum, and $\phi_{j}$ is an eigenvector with corresponding eigenvalue $1-\lambda_{j}$. It follows that 1 is an essential point of the spectrum of $T_{\Omega \backslash \bar{U}}$, so that $\left\|T_{\Omega \backslash \bar{U}}\right\|_{e}=1$. Therefore, by [Çş18, Lemma 1] it follows that $\left\|R_{\Omega \backslash \bar{U}}\right\|_{e}=1$, and consequently, we have $\left\|R_{\Omega \backslash \bar{U}}\right\|=1$.

We prove part (2) of Theorem 1 in a slightly stronger form below in Proposition 9 ,

Proposition 9. Let $\Omega$ be a domain in $\mathbb{C}^{n}$ with nontrivial Bergman space and $U$ be a non-empty open subset of $\Omega$ such that $\Omega \backslash \bar{U} \neq \emptyset$ and $\partial U$ has zero Lebesgue measure. Then the following are equivalent 
(1) $\left\|R_{U}\right\|<1$,

(2) $R_{\Omega \backslash \bar{U}}$ has closed range,

(3) $T_{\Omega \backslash \bar{U}}$ is a linear homeomorphism.

Proof. Assume (1), i.e., $\left\|R_{U}\right\|<1$. Now $\left\|T_{U}\right\|=\left\|R_{U}\right\|^{2}$ is the maximum of the spectrum of $T_{U}$, so that $\operatorname{Spec}\left(T_{U}\right) \subset\left[0,\left\|R_{U}\right\|^{2}\right]$. From (16) we see that $\operatorname{Spec}\left(T_{\Omega \backslash \bar{U}}\right) \subset\left[1-\left\|R_{U}\right\|^{2}, 1\right]$. Then, by the spectral theorem (see, for example, [RSN90, 275f.]) we have for each $f \in A^{2}(\Omega)$ we have

$$
\left\langle T_{\Omega \backslash \bar{U}} f, f\right\rangle_{\Omega} \geq\left(1-\left\|R_{U}\right\|^{2}\right)\|f\|_{\Omega}^{2},
$$

which implies that

$$
\left\|R_{\Omega \backslash \bar{U}} f\right\|_{\Omega \backslash \bar{U}} \geq \sqrt{1-\left\|R_{U}\right\|^{2}}\|f\|_{\Omega}
$$

Hence, $R_{\Omega \backslash \bar{U}}$ has closed range, i.e. (2).

Assume (2) now. Thanks to Proposition 1, we already know that $T_{U}$ is injective and has dense range, so it suffices to show that $T_{\Omega \backslash \bar{U}}$ has closed range. Now since by hypothesis, $R_{\Omega \backslash \bar{U}}$ has closed range and we know it is injective, it follows from the open mapping theorem that there is a a $C>0$ such that $\left\|R_{\Omega \backslash \bar{U}} f\right\| \geq C\|f\|$ for all $f \in A^{2}(\Omega)$. This is equivalent to the condition that

$$
\left\langle T_{\Omega \backslash \bar{U}} f, f\right\rangle_{\Omega}=\left\langle\left(R_{\Omega \backslash \bar{U}}\right)^{*} R_{\Omega \backslash \bar{U}} f, f\right\rangle_{\Omega} \geq C^{2}\|f\|_{\Omega}^{2} .
$$

Since $T_{\Omega \backslash \bar{U}}$ is self-adjoint, this means that $\left\|T_{\Omega \backslash \bar{U}} f\right\| \geq C\|f\|$ for each $f \in A^{2}(\Omega)$, which shows that $T_{\Omega \backslash \bar{U}}$ has closed range, thus completing the proof of (3).

Now suppose (3) holds, so that $T_{\Omega \backslash \bar{U}}$ has closed range, which is equivalent to (18). It follows that $\operatorname{Spec}\left(T_{\Omega \backslash \bar{U}}\right) \subset[C, 1]$. Using (16) we see that $\operatorname{Spec}\left(T_{U}\right) \subset[0,1-C]$. Consequently,

$$
\left\|R_{U}\right\|^{2}=\left\|T_{U}\right\| \leq 1-C<1 .
$$

Hence, the proof of Proposition 9 is complete.

\section{Norm estimates on tangent domains and proof of Theorem 2}

5.1. Planar situation. In the case when the dimension $n=1$, the following result gives the norm of the restriction operator on tangent domains.

Proposition 10. Let $\Omega$ be a bounded domain in $\mathbb{C}$ and $U$ be a non-empty open subset of $\Omega$. Suppose that there is a $p \in \partial \Omega \cap \partial U$ near which $\partial \Omega$ and $\partial U$ are $C^{1}$-smooth. Then $\left\|R_{U}\right\|=1$.

Note that thanks to Proposition 5 this means that $R_{U}$ is non-compact.

Proof. Without loss of generality we assume that the origin is a boundary point and the negative $x$-axis is the outward normal at the origin. Then for $\varepsilon>0$ small we can find $\delta>0$ so that

$$
U_{\varepsilon, \delta} \subset U \cap \mathbb{D}_{\delta} \subset \Omega \cap \mathbb{D}_{\delta} \subset V_{\varepsilon, \delta} .
$$

where $\mathbb{D}_{\delta}=\{z \in \mathbb{C}:|z|<\alpha\}$

$$
U_{\varepsilon, \delta}=\left\{r e^{i \theta}:|\theta|<\frac{\pi}{2}-\varepsilon, 0<r<\delta\right\}, \text { and } V_{\varepsilon, \delta}=\left\{r e^{i \theta}:|\theta|<\frac{\pi}{2}+\varepsilon, 0<r<\delta\right\} .
$$

Let us choose $f_{j}(z)=a_{j} z^{-\alpha_{j}}$ where $\alpha_{j}=1-2^{-j}$ and $a_{j}=\delta^{\alpha_{j}-1} \sqrt{\frac{2-2 \alpha_{j}}{\pi+2 \varepsilon}}$. Then one can compute that $a_{j} \rightarrow 0$ and $\left\|f_{j}\right\|_{V_{\varepsilon, \delta}}^{2}=1$. The fact that $a_{j} \rightarrow 0$ implies that $f_{j} \rightarrow 0$ on any compact set away from the 
origin. That is, the mass of $f_{j}$ accumulates near the origin as $j \rightarrow \infty$. Furthermore, one can compute that $\left\|f_{j}\right\|_{U_{\varepsilon, \delta}}^{2}=\frac{\pi-2 \varepsilon}{\pi+2 \varepsilon}$. Hence for every $\varepsilon>0$ there exists $j$ such that

$$
\frac{\pi-2 \varepsilon}{\pi+2 \varepsilon} \leq\left\|f_{j}\right\|_{U}^{2} \leq\left\|f_{j}\right\|_{\Omega}^{2} \leq 1+\varepsilon
$$

Therefore, $\left\|R_{U}\right\|=1$.

5.2. Higher dimensional situation. The following example and Theorem 2 , show that the situation in higher dimensions is different.

Example 3. Let $\Omega \subset \mathbb{C}^{2}$ be the bidisc, $0<\rho_{1}, \rho_{2}<1$, and

$$
U=U_{1} \times U_{2}=\left\{z \in \Omega:\left|z_{1}\right|<\rho_{1}, \rho_{2}<\left|z_{2}\right|<1\right\}
$$

be the product of the disc $U_{1}=\left\{z \in \mathbb{C}:|z|<\rho_{1}\right\}$ and the annulus $U_{2}=\left\{z \in \mathbb{C}: \rho_{2}<|z|<1\right\}$. The eigenvalues of the associated Toeplitz operator $T_{U}$ of the restriction operator $R_{U}: A^{2}(\Omega) \rightarrow A^{2}(U)$ can be found using formula (12), which are given by $\left(\alpha \in \mathbb{Z}_{+}^{2}\right)$

$$
\lambda_{\alpha}=\frac{\left\|z^{\alpha}\right\|_{U}^{2}}{\left\|z^{\alpha}\right\|_{\Omega}^{2}}=\rho_{1}^{2 \alpha_{1}+2}\left(1-\rho_{2}^{2 \alpha_{2}+2}\right) .
$$

Consequently, we have by (13)

$$
\left\|R_{U}\right\|=\sup _{\alpha \in \mathbb{Z}_{+}^{2}} \sqrt{\rho_{1}^{2 \alpha_{1}+2}\left(1-\rho_{2}^{2 \alpha_{2}+2}\right)}=\rho_{1} .
$$

This example shows that Proposition 10 does not hold in higher dimensions, and in fact it is possible for $\Omega$ and $U$ to share an open subset of the boundary and still have $\left\|R_{U}\right\|<1$.

Note that $V=\Omega \backslash \bar{U}$ is the well-known Hartogs figure, and it is not difficult to see that the map $R_{V}: A^{2}(\Omega) \rightarrow A^{2}(V)$ is surjective.

We close this section with the proof of Theorem 2 ,

Proof of Theorem Q. Without any loss of generality, we can assume that $R=1$. Taking $f \equiv 1$, we see that

$$
\|f\|_{\Omega}^{2}=\int_{\Omega} 1 d V=\operatorname{Vol}(\Omega), \text { and }\|f\|_{U}^{2}=\int_{U} 1 d V=\operatorname{Vol}(U) .
$$

Then we have $\frac{\|f\|_{U}^{2}}{\|f\|_{\Omega}^{2}}=r^{2 n} \leq\left\|R_{U}\right\|^{2}$, which establishes the lower bound in (2)). The fact that this norm is attained when $U$ and $\Omega$ are concentric follows from (15).

When $n=1$, the right hand side of (2) is 1 , so there is nothing to prove. Consequently, we will assume $n \geq 2$. We denote the coordinates of $\mathbb{C}^{n}$ by $(z, w)$, where $z \in \mathbb{C}$ and $w \in \mathbb{C}^{n-1}$. After a coordinate change given by unitary rotation and translation, we may suppose that

$$
\Omega=\left\{(z, w) \in \mathbb{C} \times \mathbb{C}^{n-1}:|z-1|^{2}+|w|^{2}<1\right\},
$$

and

Clearly

$$
U=\left\{(z, w) \in \mathbb{C} \times \mathbb{C}^{n-1}:|z-\delta|^{2}+|w|^{2}<r^{2}\right\}
$$

$$
r \leq \delta \leq 1
$$

Denoting by $\pi$ the projection from $\mathbb{C}^{n}$ to $\mathbb{C}$ given by $(z, w) \rightarrow z$, we see that $\pi(U)=\{z \in \mathbb{C}:|z-\delta|<r\}$, which is a smaller disc contained in the disc $\pi(\Omega)=\{z \in \mathbb{C}:|z-1|<1\}$. For $z \in \pi(U)$, we note that $\pi^{-1}(z) \cap U=\{z\} \times \Delta_{U}(z)$, where $\Delta_{U}(z)$ is the ball in $\mathbb{C}^{n-1}$ defined by

$$
\Delta_{U}(z)=B_{\mathbb{C}^{n-1}}\left(0, \sqrt{r^{2}-|z-\delta|^{2}}\right)=\left\{w \in \mathbb{C}^{n-1}:|w|^{2}<r^{2}-|z-\delta|^{2}\right\} .
$$


Similarly, for a $z$ in the unit disc, let $\pi^{-1}(z) \cap \Omega=\{z\} \times \Delta_{\Omega}(z)$, where $\Delta_{\Omega}(z)$ is the ball in $\mathbb{C}^{n-1}$ given by

$$
\Delta_{\Omega}(z)=B_{\mathbb{C}^{n-1}}\left(0, \sqrt{1-|z-1|^{2}}\right)=\left\{w \in \mathbb{C}^{n-1}:|w|^{2}<1-|z-1|^{2}\right\} .
$$

With this notation, we have for any integrable function $u$ the formulas

$$
\int_{\Omega} u d V=\int_{\pi(\Omega)}\left(\int_{\Delta_{\Omega}(z)} u(z, w) d V(w)\right) d V(z) \text { and } \int_{U} u d V=\int_{\pi(U)}\left(\int_{\Delta_{U}(z)} u(z, w) d V(w)\right) d V(z),
$$

by representing the integral as a repeated integral.

For notational clarity, for $z$ in the disc $\pi(U)$, let $S(z)=R_{\Delta_{U}(z)}$ denote the restriction operator

$$
S(z)=R_{\Delta_{U}(z)}: A^{2}\left(\Delta_{\Omega}(z)\right) \rightarrow A^{2}\left(\Delta_{U}(z)\right) .
$$

Since $\Delta_{\Omega}(z)$ and $\Delta_{U}(z)$ are concentric balls in $\mathbb{C}^{n-1}$, it follows from (15) that

$$
\|S(z)\|=\left(\frac{r^{2}-|z-\delta|^{2}}{1-|z-1|^{2}}\right)^{\frac{n-1}{2}} .
$$

We have, for each $f \in A^{2}(\Omega)$

$$
\begin{aligned}
\|f\|_{U}^{2} & =\int_{\pi(U)}\left(\int_{\Delta_{U}(z)}|f(z, w)|^{2} d V(w)\right) d V(z) \\
& =\int_{\pi(U)}\|f(z, \cdot)\|_{\Delta_{U}(z)}^{2} d V(z) \\
& \leq \int_{\pi(U)}\|S(z)\|^{2}\|f(z, \cdot)\|_{\Delta_{\Omega}(z)}^{2} d V(z) \\
& \leq \sup _{z \in \pi(U)}\|S(z)\|^{2} \int_{\pi(U)}\left(\int_{\Delta_{\Omega}(z)}|f(z, w)|^{2} d V(w)\right) d V(z) \\
& <\sup _{z \in \pi(U)}\|S(z)\|^{2} \int_{\pi(\Omega)}\left(\int_{\Delta_{\Omega}(z)}|f(z, w)|^{2} d V(w)\right) d V(z) \\
& =\left(\sup _{|z-\delta|<r}\|S(z)\|^{2}\right)\|f\|_{\Omega}^{2} .
\end{aligned}
$$

So by (20) we have

$$
\left\|R_{U}\right\| \leq \sup _{|z-\delta|<r}\|S(z)\|=\sup _{|z-\delta|<r}\left(\frac{r^{2}-|z-\delta|^{2}}{1-|z-1|^{2}}\right)^{\frac{n-1}{2}} .
$$

Notice now that thanks to (19), we have

$$
\left(\delta^{2}-r^{2}\right)+(1-\delta)|z|^{2} \geq 0 .
$$

Since we can write

it follows that

$$
r^{2}-|z-\delta|^{2}=\delta\left(1-|z-1|^{2}\right)-\left(\left(\delta^{2}-r^{2}\right)+(1-\delta)|z|^{2}\right)
$$

$$
\frac{r^{2}-|z-\delta|^{2}}{1-|z-1|^{2}} \leq \delta
$$

for each $z$ such that $1-|z-1|^{2}>0$ (and therefore for $z$ in the smaller set $r^{2}-|z-\delta|^{2}>0$ ). It now follows that

$$
\left\|R_{U}\right\| \leq \delta^{\frac{n-1}{2}}
$$


thus establishing the upper bound in (2).

To complete the proof, we need to show that if $\delta=r$, then we have $\left\|R_{U}\right\| \geq r^{\frac{n-1}{2}}$, for $n \geq 1$. Note that since the disc $\pi(\Omega)=\{z \in \mathbb{C}:|z-1|<1\}$ is contained in the right half plane, for each $\gamma>0$, we can define a branch of $z^{-\gamma}$ on $\Omega$. Let $f_{\gamma}$ be such a branch, normalized for uniqueness by the condition $f_{\gamma}(1)=1$. Observe that

$$
\begin{aligned}
\left\|f_{\gamma}\right\|_{U}^{2} & =\int_{\pi(U)} \int_{\Delta_{U}(z)}\left|f_{\gamma}(z, w)\right|^{2} d V(w) d V(z) \\
& =\int_{\{|z-r|<r\}}|z|^{-2 \gamma} \operatorname{Vol}\left(B_{\mathbb{C}^{n-1}}\left(0, \sqrt{r^{2}-|z-r|^{2}}\right)\right) d V(z) \\
& =\operatorname{Vol}\left(B_{\mathbb{C}^{n-1}}(0,1)\right) \int_{\{|z-r|<r\}}|z|^{-2 \gamma}\left(r^{2}-|z-r|^{2}\right)^{n-1} d V(z),
\end{aligned}
$$

where volumes of zero-dimensional balls are defined to be 1 . We now make a change of variables in the above integral to a new variable $w$, related to $z$ by

$$
z=r w
$$

so that by the change of variables formula, the above integral becomes

$$
\begin{aligned}
\left\|f_{\gamma}\right\|_{U}^{2} & =\operatorname{Vol}\left(B_{\mathbb{C}^{n-1}}(0,1)\right) \int_{\{|w-1|<1\}} r^{-2 \gamma}|w|^{-2 \gamma} r^{2(n-1)}\left(1-|w-1|^{2}\right)^{n-1} r^{2} d V(w) \\
& =r^{2 n-2 \gamma} \operatorname{Vol}\left(B_{\mathbb{C}^{n-1}}(0,1)\right)\left(\int_{\{|w-1|<1\}}|w|^{-2 \gamma}\left(1-|w-1|^{2}\right)^{n-1} d V(w)\right) \\
& =r^{2(n-\gamma)}\left\|f_{\gamma}\right\|_{\Omega}^{2} .
\end{aligned}
$$

Therefore, for each $\gamma$ such that $f_{\gamma} \in A^{2}(\Omega)$ we have $\left\|R_{U}\right\| \geq r^{n-\gamma}$. We now claim that if $\gamma<\frac{n+1}{2}$, then $f_{\gamma} \in A^{2}(\Omega)$. Assuming the claim, we conclude that

$$
\left\|R_{U}\right\| \geq \sup _{\gamma<\frac{n+1}{2}} r^{n-\gamma}=r^{\frac{n-1}{2}}
$$

which completes the proof of the result modulo the claim. To prove the claim, it suffices to show that the integral in (21) is finite if $\gamma<\frac{n+1}{2}$. In fact, since

$$
1-|w-1|^{2}=2 \operatorname{Re} w-|w|^{2} \leq 2 \operatorname{Re} w
$$

it follows that it suffices to show that the integral

$$
\int_{\{|w-1|<1\}}|w|^{-2 \gamma}(\operatorname{Re} w)^{n-1} d V(w)
$$

is finite. Switching to polar coordinates $w=\rho e^{i \theta}$, this integral becomes

$$
\begin{aligned}
\int_{\theta=-\frac{\pi}{2}}^{\frac{\pi}{2}} \int_{\rho=0}^{2 \cos \theta} \rho^{-2 \gamma}(\rho \cos \theta)^{n-1} \rho d \rho d \theta & =\int_{-\frac{\pi}{2}}^{\frac{\pi}{2}}(\cos \theta)^{n-1}\left(\int_{0}^{2 \cos \theta} \rho^{n-2 \gamma} d \rho\right) d \theta \\
& =\left.\int_{-\frac{\pi}{2}}^{\frac{\pi}{2}}(\cos \theta)^{n-1} \frac{\rho^{n-2 \gamma+1}}{n-2 \gamma+1}\right|_{0} ^{2 \cos \theta} d \theta
\end{aligned}
$$

We note that $\gamma<\frac{n+1}{2}$ implies that the integral $\int_{0}^{2 \cos \theta} \rho^{n-2 \gamma} d \rho$ converges at the endpoint 0 as $n-2 \gamma>-1$. Therefore, up to some irrelevant multiplying constants, the last integral above becomes

$$
\int_{-\frac{\pi}{2}}^{\frac{\pi}{2}}(\cos \theta)^{n-1}(\cos \theta)^{n-2 \gamma+1} d \theta=\int_{-\frac{\pi}{2}}^{\frac{\pi}{2}}(\cos \theta)^{2(n-\gamma)} d \theta
$$


Since $\gamma<\frac{n+1}{2}$, it follows that $2(n-\gamma)>n-1 \geq 0$, which shows that the integrand in the above integral is continuous. This shows that $f_{\gamma} \in A^{2}(\Omega)$.

\section{Proof of Theorems 3 and 4}

6.1. Two results of N. Vasilevski. We will deduce Theorems 3 and 4 as special cases of the following determination of spectra of Toeplitz operators on the Bergman space of the upper half plane $\mathbb{H}=\{z \in$ $\mathbb{C} \mid \operatorname{Im}(z)>0\}$. Recall that given a function $\psi \in L^{\infty}(\mathbb{H})$, the Toeplitz operator $T_{\psi}$ with symbol $\psi$ is the bounded linear operator on $A^{2}(\mathbb{H})$ defined as

$$
T_{\psi} f=P(\psi f)
$$

where $P: L^{2}(\mathbb{H}) \rightarrow A^{2}(\mathbb{H})$ is the Bergman projection on $A^{2}(\mathbb{H})$. Recall also that given a measure space $(X, \mathcal{F}, \mu)$ and a function $\gamma \in L^{\infty}(\mu)$, the multiplication operator $M_{\gamma}$ on $L^{2}(\mu)$ with multiplier $\gamma$ is the bounded operator defined by multiplication by $\gamma$ :

$$
M_{\gamma} f=\gamma f \text {. }
$$

Theorem 6 ([Vas08, Theorem 5.2.1]). Let $\mathbb{R}^{+}=(0, \infty), \psi \in L^{\infty}(\mathbb{H})$, and $a \in L^{\infty}\left(\mathbb{R}^{+}\right)$such that $\psi(z)=$ $a(\operatorname{Im} z)$ for all $z \in \mathbb{H}$. Then the Toeplitz operator $T_{\psi}$ on $A^{2}(\mathbb{H})$ is unitarily equivalent to the multiplication operator on $L^{2}\left(\mathbb{R}^{+}\right)$with multiplier $\gamma$ given by

$$
\gamma(x)=\int_{\mathbb{R}^{+}} a\left(\frac{\eta}{2 x}\right) e^{-\eta} d \eta .
$$

Theorem 7 ([Vas08, Theorem 7.2.1]). Let $\psi \in L^{\infty}(\mathbb{H})$ and $a \in L^{\infty}(0, \pi)$ such that $\psi\left(r e^{i \theta}\right)=a(\theta)$ for all $0<\theta<\pi$. Then the Toeplitz operator $T_{\psi}$ on $A^{2}(\mathbb{H})$ is unitarily equivalent to the multiplication operator on $L^{2}(\mathbb{R})$ with multiplier $\gamma$ given by

$$
\gamma(\lambda)=\frac{2 \lambda}{1-e^{-2 \pi \lambda}} \int_{0}^{\pi} a(\theta) e^{-2 \lambda \theta} d \theta
$$

Such remarkable explicit determination of the spectrum of a Toeplitz operator is terms of the symbol is quite rare and can be achieved only on very symmetric special cases as above.

6.2. Mapping to the upper half-plane. In order to apply Theorems 6 and 7 to the proofs of Theorems 3 and 4, respectively, we begin by mapping the unit disc $\mathbb{D}$ conformally to the upper half plane $\mathbb{H}$. Thanks to Proposition 2 we know that the spectrum of $T_{U}$ is invariant under biholomorphisms of the ambient domain, so we can use this upper half-plane model to compute the spectrum.

Consider, as in Theorem 3, part 2, a horocyclic strip in the unit disc, where the outer and inner bounding horocycles have Euclidean radii $\rho_{2}$ and $\rho_{1}$ respectively. After a rotation of the unit disc, we may assume that the point of contact is at $1 \in \partial \mathbb{D}$. By the standard conformal map of $\mathbb{D}$ to $\mathbb{H}$ given by

$$
z \mapsto i \frac{1+z}{1-z}
$$

the bounding horocycle $\left\{z \in \mathbb{C}:\left|z-\left(1-\rho_{j}\right)\right|=\rho_{j}\right\}$ is mapped to a Euclidean straight line in the upper half plane parallel to the real axis, given by $\left\{z \in \mathbb{C}: \operatorname{Im} z=\frac{1}{\rho_{j}}-1\right\}$. Therefore, the horocyclic strip bounded by Euclidean circles of radii $0<\rho_{1}<\rho_{2}<1$ is therefore mapped into the horizontal strip in the upper half plane given by

$$
\left\{z \in \mathbb{C}: \frac{1}{\rho_{2}}-1<\operatorname{Im} z<\frac{1}{\rho_{1}}-1\right\} .
$$

Applying the dilation of the upper half plane given by $z \mapsto \frac{z}{\frac{1}{\rho_{2}}-1}$, this is mapped onto the horizontal strip

$$
V_{\alpha}=\{z \in \mathbb{C}: 1<\operatorname{Im} z<\alpha\}
$$



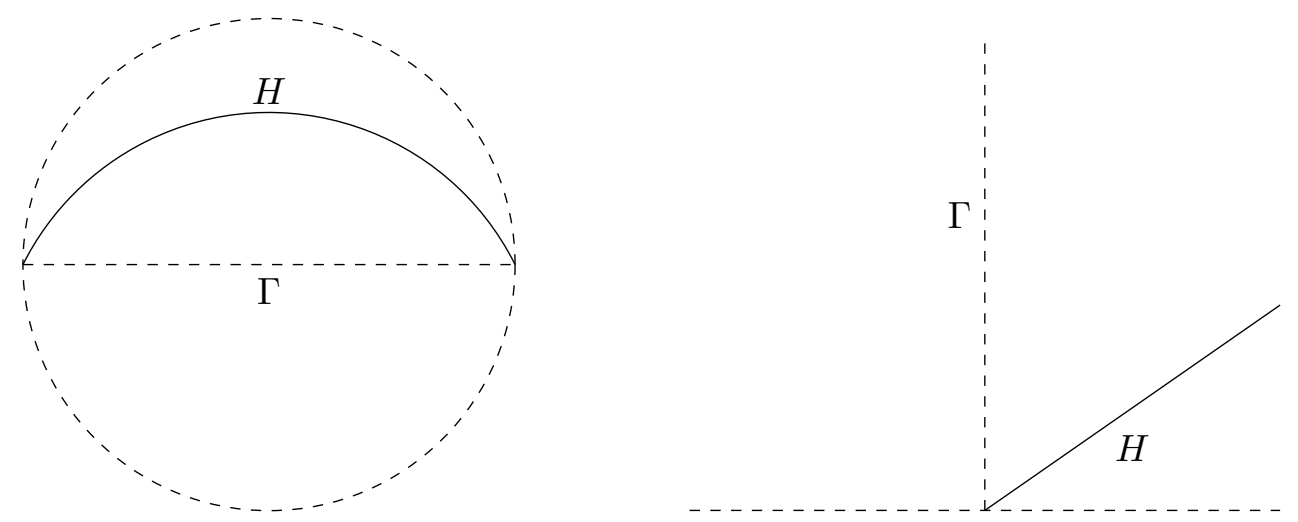

Figure 4. Hypercycles in the disc and the upper half-plane

where $\alpha$ is as in (3). The full horodisc corresponds to the limiting situation of $\rho_{1} \rightarrow 0$, and therefore can be mapped to the half-plane

$$
V_{\infty}=\{z \in \mathbb{C}: \operatorname{Im} z>1\} .
$$

We now consider the mapping of hypercycles and lunes and crescents determined by them. Let $H$ be a hypercycle in the Poincaré disc with axis $\Gamma$. Recall that by definition this means that $H$ is a connected curve consisting of points at a fixed hyperbolic distance from $\Gamma$. After applying an automorphism of the unit disc, the geodesic $\Gamma$ is mapped onto the interval $(-1,1)$ on the real axis. The hypercycle $H$ is mapped to an arc of a circle passing through the points \pm 1 (see Figure 44). Under the conformal map (24), -1 is mapped to 0,1 is mapped to $\infty$ and therefore the diameter $\Gamma=(-1,1)$ is mapped to the positive imaginary axis. It follows that the hypercycle $H$ (which is an arc of an Euclidean circle in the complex plane) is mapped to a rectilinear ray in the upper half plane $\mathbb{H}$ passing through 0 . Therefore, a hypercyclic lune is represented in the upper half plane by a wedge of the form

$$
W(\alpha, \beta)=\left\{r e^{i \theta} \in \mathbb{H} \mid r>0 \text { and } \alpha<\theta<\beta\right\},
$$

where $0<\alpha<\beta<\pi$. Similarly, a hypercyclic crescent takes the form $W(\alpha, \pi)$ or $W(0, \beta)$.

Proof of Theorem 3. Thanks to Proposition 2 and the conformal mappings constructed in Section 6.2, the spectrum of a horodisc in $\mathbb{D}$ coincides with that of the operator $T_{V_{\infty}}$ on $A^{2}(\mathbb{H})$ where $V_{\infty}=\{z \in \mathbb{C}: \operatorname{Im} z>$ $1\}$. It follows easily from Theorem [6, the spectrum of $T_{V_{\infty}}$ is the interval

$$
\left[\inf _{x \in \mathbb{R}^{+}} \gamma(x), \sup _{x \in \mathbb{R}^{+}} \gamma(x)\right]
$$

where $\gamma$ is as in (22), with $a=\chi_{[1, \infty)}$, the characteristic function of the interval $[1, \infty)$. Therefore, for $x>0$, we have

$$
\gamma(x)=\int_{\frac{\eta}{2 x}>1} e^{-\eta} d \eta=\int_{2 x}^{\infty} e^{-\eta} d \eta=e^{-2 x} .
$$

Therefore $\inf _{x \in \mathbb{R}^{+}} \gamma(x)=0$ and $\sup _{x \in \mathbb{R}^{+}} \gamma(x)=1$, and part (1) of Theorem 3 follows.

For part (2), note that the spectrum is still given by (26), provided we define $\gamma$ in (22) with $a=\chi_{[1, \alpha]}$. Therefore, in this case, for each $x>0$.

$$
\gamma(x)=\int_{1<\frac{\eta}{2 x}<\alpha} e^{-\eta} d \eta=\int_{2 x}^{2 x \alpha} e^{-\eta} d \eta=e^{-2 x}-e^{-2 x \alpha}
$$


But as $\lim _{x \rightarrow \infty}\left(e^{-2 x}-e^{-2 x \alpha}\right)=0$, it follows that $\inf _{x \in \mathbb{R}^{+}} \gamma(x)=0$. To find the supremum, note that $\gamma^{\prime}(x)=-2 e^{-2 x}+2 \alpha e^{-2 x \alpha}$, so that the only critical point of $\gamma$ is given by

$$
x_{\text {crit }}=\frac{1}{2} \frac{\ln \alpha}{\alpha-1} .
$$

Note also that $\lim _{x \rightarrow 0} \gamma(x)=0$, and we must have $0 \leq \gamma \leq 1$ (since the range of $\gamma$ coincides with the spectrum of $T_{V_{\alpha}}$, which we know to be a subset of $[0,1]$.) So it follows that the critical point $x_{\text {crit }}$ is in fact a point of global maximum of $\gamma$ on $\mathbb{R}^{+}$. Therefore

$$
\sup _{x \in \mathbb{R}^{+}} \gamma(x)=\gamma\left(x_{\text {crit }}\right)=\alpha^{-\frac{1}{\alpha-1}}-\alpha^{-\frac{\alpha}{\alpha-1}},
$$

which completes the proof of Theorem 3 .

Proof of Theorem 4. By biholomorphic invariance, it suffices to consider the operator $T_{W(\alpha, \beta)}$ on $A^{2}(\mathbb{H})$. Thanks to Theorem[7, the spectrum of $T_{W(\alpha, \beta)}$ coincides with the closure of range of the function $\gamma: \mathbb{R} \rightarrow \mathbb{R}$ given by

$$
\gamma(\lambda)=\frac{2 \lambda}{1-e^{-2 \pi \lambda}} \int_{\alpha}^{\beta} e^{-2 \lambda \theta} d \theta=\frac{e^{-2 \lambda \alpha}-e^{-2 \lambda \beta}}{1-e^{-2 \pi \lambda}}=\frac{\xi^{b}-\xi^{a}}{\xi-1}
$$

where $\xi=\xi(\lambda)=e^{-2 \pi \lambda}, a=\frac{\alpha}{\pi}, b=\frac{\beta}{\pi}$, so that $0 \leq a<b \leq 1$. Note that $\xi \rightarrow 0$ as $\lambda \rightarrow \infty$ and $\xi \rightarrow \infty$ as $\lambda \rightarrow-\infty$.

First consider the situation of a hypercyclic crescent represented in $\mathbb{H}$ by $W(\alpha, \pi)$, where $0<\alpha<\pi$. Then, in (27) we have $b=1,0<a<1$, and $\gamma=\frac{\xi-\xi^{a}}{\xi-1}$. So $\lambda \rightarrow \infty$ implies that $\xi \rightarrow 0$ which, in turn implies that $\gamma \rightarrow 0$. On the other hand, $\lambda \rightarrow-\infty$ implies that $\xi \rightarrow \infty$ and hence $\gamma \rightarrow 1$. Therefore we have

$$
\operatorname{spec}\left(T_{W(\alpha, \pi)}\right)=\left[\sup _{\lambda \in \mathbb{R}} \gamma(\lambda), \inf _{\lambda \in \mathbb{R}} \gamma(\lambda)\right]=[0,1] .
$$

The other possibility for a hypercyclic crescent is to be represented in $\mathbb{H}$ by the wedge $W(0, \beta)$, where $0<\beta<1$. Then in (27), we have $a=0$ and $0<b<1$. So we have $\gamma=\frac{\xi^{b}-1}{\xi-1}$. Therefore as $\lambda \rightarrow \infty$ we have $\xi \rightarrow 0$ and $\gamma \rightarrow 1$. Similarly as $\lambda \rightarrow-\infty$ we have $\xi \rightarrow \infty$ and $\gamma \rightarrow 0$. This shows again that the spectrum of $T_{W(0, \beta)}$ equals to [0,1]. This concludes the proof of Part (1) of Theorem 4 .

For part (2) of Theorem 4 , if $0<a<b<1$, then the function

$$
\frac{\xi^{b}-\xi^{a}}{\xi-1}
$$

goes to 0 as either $\xi \rightarrow \infty$ or $\xi \rightarrow 0$ (i.e., $\lambda \rightarrow \pm \infty$ ). It follows that the spectrum of $T_{W(\alpha, \beta)}$ is of the form $[0, c(\alpha, \beta)]$, where

$$
c(\pi a, \pi b)=c(\alpha, \beta)=\left\|T_{W(\alpha, \beta)}\right\|=\sup _{0<\xi<\infty} \frac{\xi^{b}-\xi^{a}}{\xi-1} .
$$

The function $c(\alpha, \beta)$ cannot be expressed in closed form. But note that for fixed $0<\alpha<\pi$ as $\beta \rightarrow \alpha^{+}$ clearly $c(\alpha, \beta) \rightarrow 0$ and as $\beta \rightarrow \pi, c(\alpha, \beta) \rightarrow 1$, since as $b \rightarrow a$, the function $\xi \mapsto \frac{\xi^{b}-\xi^{a}}{\xi-1}$ converges uniformly to 0 on compact sets, and as $b \rightarrow 1$, it converges uniformly to 1 on compact sets. To complete the proof we need to show that $c(\alpha, \beta)=\left\|T_{W(\alpha, \beta)}\right\|<1$ This follows from an elementary computation which is given below as a separate Lemma.

Lemma 1. Let $0<a<b<1$. Then

$$
c(\pi a, \pi b)=\sup _{0<\xi<\infty} \frac{\xi^{b}-\xi^{a}}{\xi-1}<1 .
$$


Proof. Let $f(\xi)=\frac{\xi^{b}-\xi^{a}}{\xi-1}$. As $\xi \rightarrow 0$, we have $f(\xi) \rightarrow 0$. Therefore, there is a $\delta>0$ such that

$$
\sup _{0<\xi \leq \delta} \frac{\xi^{b}-\xi^{a}}{\xi-1}<\frac{1}{2}
$$

Since $0<a<b<1$, we have, for $\delta<\xi<1$ that

$$
\delta<\xi<\xi^{b}<\xi^{a}<1 .
$$

and therefore that

$$
f(\xi)=\frac{1-\xi^{b}}{1-\xi}-\frac{1-\xi^{a}}{1-\xi}<\frac{1-\xi^{b}}{1-\xi}<1 .
$$

Combined with the facts that $\lim _{\xi \rightarrow \delta} f(\xi)=f(\delta)<\frac{1}{2}$ and

$$
\lim _{\xi \rightarrow 1} f(\xi)=\lim _{\xi \rightarrow 1} \frac{b \xi^{b-1}-a \xi^{a-1}}{1}=b-a<b<1,
$$

we obtain that

$$
\sup _{\delta \leq \xi \leq 1} \frac{\xi^{b}-\xi^{a}}{\xi-1}<1
$$

Furthermore, if $\xi>1$ then $1<\xi^{a}<\xi^{b}<\xi$. So we have

$$
f(\xi)=\frac{\xi^{b}-1}{\xi-1}-\frac{\xi^{a}-1}{\xi-1}<\frac{\xi^{b}-1}{\xi-1}<1 .
$$

Therefore, $f(\xi)<1$ for each $\xi \in[1, \infty)$. Further, since $\lim _{\xi \rightarrow \infty} f(\xi)=0$, it follows that

$$
\sup _{1 \leq \xi<\infty} \frac{\xi^{b}-\xi^{a}}{\xi-1}<1 .
$$

Combining (28), (29), (30) the result follows.

\subsection{Some examples and comments.}

6.3.1. Isospectrality of hypercyclic crescents and horodiscs. Theorems 3 and 4 show that a horodisc and a hypercyclic crescent are isospectral, both having spectrum $[0,1]$. But they are not congruent to each other (i.e., there is no automorphism of $\mathbb{D}$ which maps a hypercyclic crescent to a horodisc). Indeed, the boundary relative to the unit disc of a hypercyclic crescent is a hypercycle, whereas that of a horodisc is a horocycle. But the geodesic curvature of a hypercycle is less than $2 \sqrt{\pi}$ in absolute value, whereas that of a horocycle is equal to $2 \sqrt{\pi}$ in absolute value (see [Vas08, Section 11.6, pg 282]). Since the automorphisms are isometries of the Poincaré metric, all quantities defined in terms of the metric (such as curvature) are invariant. Therefore, horocycles and hypercycles are not congruent.

6.3.2. Isospectral hypercyclic lunes and horocyclic strips. Another example of such isospectral but noncongruent subdomains of the disc is obtained by looking at two hypercyclic crescents $U_{1}$ and $U_{2}$ with coaxial but distinct bounding hypercycles $H_{1}$ and $H_{2}$, since $H_{1}$ and $H_{2}$ have different geodesic curvatures with respect to the hyperbolic metric (see [Vas08, Theorem 11.6.2]). Note also that given any $0<c<1$, there is a horocyclic strip and a hypercyclic lune (which are non-congruent thanks to the curvature argument above), such that each has spectrum $[0, c]$. This follows from a closer analysis of the formulas in part (ii) of each of Theorems 3 and 4 . 
6.3.3. Examples of closed range. Consider a horocyclic strip $H$ in the disc $\mathbb{D}$, like the shaded region in Figure 1. Then the complement $U$ of $H$ can be written as a disjoint union

$$
U=\mathbb{D} \backslash H=U_{1} \cup U_{2},
$$

where $U_{1}$ is a horodisc and $U_{2}$ is the complement of a horodisc (therefore $U_{2}$ is bounded by a horocycle and the unit circle.) From Proposition 8 we see that the spectrum of $U_{2}$ relative to $\mathbb{D}$ is the interval $[0,1]$. Since the spectrum of $H$ is of the form $\left[0,\left\|T_{H}\right\|\right]$, where $0<\left\|T_{H}\right\|<1$, we see again from Proposition 8 that

$$
\operatorname{spec}\left(T_{U}\right)=\left[1-\left\|T_{H}\right\|, 1\right]
$$

so that in particular, by Corollary 9, the restriction operator on $U=U_{1} \cup U_{2}$ has closed range.

Another similar example is the complement of the hypercyclic lune $W$ in Figure 2, Since again the spectrum of $W$ relative to $\mathbb{D}$ is the interval $\left[0,\left\|T_{W}\right\|\right]$ it follows that the complement $\mathbb{D} \backslash \bar{W}$ has spectrum $\left[1-\left\|T_{W}\right\|, 1\right]$, and the restriction operator from $A^{2}(\mathbb{D})$ to $A^{2}(\mathbb{D} \backslash \bar{W})$ has closed range.

\section{Proof of Theorem 5}

We will use the following fact (see GKK11, Theorem 1.3.12]) in the proof of Theorem 5; If $\Omega$ is a bounded domain in $\mathbb{C}^{n}$, the map

$$
\begin{aligned}
\operatorname{Aut}(\Omega) \times \Omega & \rightarrow \Omega \times \Omega \\
(\phi, z) & \mapsto(\phi(z), z)
\end{aligned}
$$

is proper. Recall that to say that a continuous map between topological spaces is proper means that the inverse image of each compact subset of the codomain is compact in the domain of the map. Recall also that the topology on $\operatorname{Aut}(\Omega)$ is the natural compact-open topology.

Proof of Theorem 5. Since $G$ is a closed subset of $\operatorname{Aut}(\Omega)$, it follows from [GKK11, Theorem 1.3.12] that the restricted map

$$
\begin{aligned}
G \times \Omega & \rightarrow \Omega \times \Omega \\
(\phi, z) & \mapsto(\phi(z), z)
\end{aligned}
$$

is also proper. Let $z_{0} \in \Omega$ and consider the orbit of $p$ under $\Omega$ i.e. the set

$$
G\left(z_{0}\right)=\left\{\phi\left(z_{0}\right) \mid \phi \in G\right\} .
$$

We claim that $G\left(z_{0}\right)$ is noncompact. Indeed, if it were compact, its inverse image under the map (31) would be compact, i.e., $G \times\left\{z_{0}\right\}$ would be compact; but by hypothesis $G$ is noncompact, so we have a contradiction. Choosing $z_{0} \in U$, and noting that by hypothesis $G\left(z_{0}\right) \subset U$, we see that there is a point $q \in \partial \Omega \cap \partial U$, and a sequence of automorphisms $\left\{\Phi_{j}\right\} \subset G$ such that $\Phi_{j}\left(z_{0}\right) \rightarrow q$ as $j \rightarrow \infty$. Consider the functions $f_{j} \in A^{2}(\Omega)$ defined by

$$
f_{j}(z)=\frac{B_{\Omega}\left(z, \Phi_{j}\left(z_{0}\right)\right)}{\sqrt{B_{\Omega}\left(\Phi_{j}\left(z_{0}\right), \Phi_{j}\left(z_{0}\right)\right)}} .
$$

We begin by noting (using the reproducing property of the Bergman kernel) that

$$
\begin{aligned}
\left\|f_{j}\right\|_{A^{2}(\Omega)} & =\frac{1}{B_{\Omega}\left(\Phi_{j}\left(z_{0}\right), \Phi_{j}\left(z_{0}\right)\right)} \int_{\Omega} B_{\Omega}\left(z, \Phi_{j}\left(z_{0}\right)\right) B_{\Omega}\left(\Phi_{j}\left(z_{0}\right), z\right) d V(z) \\
& =\frac{1}{B_{\Omega}\left(\Phi_{j}\left(z_{0}\right), \Phi_{j}\left(z_{0}\right)\right)} \cdot B_{\Omega}\left(\Phi_{j}\left(z_{0}\right), \Phi_{j}\left(z_{0}\right)\right) \\
& =1 .
\end{aligned}
$$


Assume now for a contradiction that $T_{U}$ is compact, so that the restriction operator $R_{U}: A^{2}(\Omega) \rightarrow A^{2}(U)$ is also compact. Consequently, after passing to a subsequence if necessary, we may assume that the sequence $R_{U} f_{j}=\left.f_{j}\right|_{U}$ converges in the $L^{2}$-norm as $j \rightarrow \infty$ to a $g \in A^{2}(U)$. Now, note that

$$
\begin{aligned}
\int_{U}\left|f_{j}\right|^{2} d V & =\frac{1}{B_{\Omega}\left(\Phi_{j}\left(z_{0}\right), \Phi_{j}\left(z_{0}\right)\right)} \int_{U}\left|B_{\Omega}\left(z, \Phi_{j}\left(z_{0}\right)\right)\right|^{2} d V(z) \\
& =\frac{1}{B_{\Omega}\left(\Phi_{j}\left(z_{0}\right), \Phi_{j}\left(z_{0}\right)\right) \cdot\left|\operatorname{det} \Phi_{j}^{\prime}\left(z_{0}\right)\right|^{2}} \int_{U}\left|B_{\Omega}\left(z, \Phi_{j}\left(z_{0}\right)\right)\right|^{2} \cdot\left|\operatorname{det} \Phi_{j}^{\prime}\left(z_{0}\right)\right|^{2} d V(z) \\
& =\frac{1}{B_{\Omega}\left(z_{0}, z_{0}\right)} \int_{U}\left|B_{\Omega}\left(\Phi_{j}(w), \Phi_{j}\left(z_{0}\right)\right)\right|^{2} \cdot\left|\operatorname{det} \Phi_{j}^{\prime}(w)\right|^{2} \cdot\left|\operatorname{det} \Phi_{j}^{\prime}\left(z_{0}\right)\right|^{2} d V(w) \\
& =\frac{1}{B_{\Omega}\left(z_{0}, z_{0}\right)} \int_{U}\left|\frac{1}{\operatorname{det} \Phi_{j}^{\prime}(w)} \cdot B_{\Omega}\left(\Phi_{j}(w), \Phi_{j}\left(z_{0}\right)\right) \cdot \operatorname{det} \Phi_{j}^{\prime}\left(z_{0}\right)\right|^{2} d V(w) \\
& =\frac{1}{B_{\Omega}\left(z_{0}, z_{0}\right)} \int_{U}\left|B_{\Omega}\left(w, z_{0}\right)\right|^{2} d V(w) \\
& =C^{2},
\end{aligned}
$$

where $C>0$ is independent of $j$, and during the course of the computation we have used twice the transformation formula for the Bergman kernel under a biholomorphic map. Therefore, the $L^{2}$-limit $g$ of the sequence $f_{j}$ also satisfies $\|g\|_{A^{2}(U)}=C$. On the other hand, we claim that $f_{j} \rightarrow 0$ weakly as $j \rightarrow \infty$, which contradicts the fact that $f_{j}$ tends to a nonzero limit in the $L^{2}$-norm. This contradiction shows that $R_{U}$ and therefore $T_{U}$ is not compact.

To complete the proof we justify the claim that $f_{j} \rightarrow 0$ weakly as $j \rightarrow \infty$. Since $\Omega$ is smoothly bounded and pseudoconvex by a result of Catlin [Cat80, Theorem 3.2.1], $A^{\infty}(\bar{\Omega})$, the space of functions holomorphic on $\Omega$ and smooth up to the boundary, is dense in $A^{2}(\Omega)$. Fix $h \in A^{2}(\Omega)$, and let $\varepsilon>0$ be given. Then there exists $h_{\delta} \in A^{\infty}(\bar{\Omega})$ such that $\left\|h-h_{\delta}\right\|<\varepsilon$. Then

$$
\left|\left\langle h, f_{j}\right\rangle\right| \leq\left|\left\langle h-h_{\delta}, f_{j}\right\rangle\right|+\left|\left\langle h_{\delta}, f_{j}\right\rangle\right| \leq\left\|h-h_{\delta}\right\|+\left|\left\langle h_{\delta}, f_{j}\right\rangle\right|<\varepsilon+\left|\left\langle h_{\delta}, f_{j}\right\rangle\right|
$$

However, we note that $\left\langle h_{\delta}, f_{j}\right\rangle=h_{\delta}\left(\Phi_{j}\left(z_{0}\right)\right) / \sqrt{B_{\Omega}\left(\Phi_{j}\left(z_{0}\right), \Phi_{j}\left(z_{0}\right)\right)} \rightarrow 0$ as $j \rightarrow \infty$ because $B_{\Omega}(z, z) \rightarrow \infty$ as $z \rightarrow q \in \partial \Omega$ (see [JP93, Theorem 6.1.17] and [Pfl75]) and $h_{\delta}$ is bounded. Since $\varepsilon$ was arbitrary we conclude that $\lim _{z \rightarrow q}\left\langle h, f_{j}\right\rangle=0$ for any $h \in A^{2}(\Omega)$. That is, $f_{j} \rightarrow 0$ weakly as $j \rightarrow \infty$ and the proof of Theorem 5 is complete.

\section{Membership in Schatten Classes}

In this section we consider the problem of computing the Schatten $p$-norms of $R_{U}$ and $T_{U}$, provided we know the Bergman kernel $B_{\Omega}: \Omega \times \Omega \rightarrow \mathbb{C}$ of the domain $\Omega$. Let us define the functions $B_{U, \Omega}^{(p)}: \Omega \times \Omega \rightarrow \mathbb{C}$ for all $p \geq 1$, by setting $B_{U, \Omega}^{(1)}=B_{\Omega}$, the Bergman kernel of $\Omega$, and (since $T_{U}$ is a positive operator)

$$
B_{U, \Omega}^{(p)}(., z)=T_{U}^{p-1} B_{\Omega}(., z)
$$

for $p>1$ and $z \in \Omega$. We note that $B_{U, \Omega}^{(p)}(., z) \in A^{2}(\Omega)$ for all $p \geq 1$ and $z \in \Omega$. We have the following formula to compute the Schatten norms of $R_{U}$ and $T_{U}$.

Proposition 11. Let $\Omega$ be a domain in $\mathbb{C}^{n}$ with nontrivial Bergman space, $U$ be a non-empty open subset of $\Omega$, and $p>0$. Then $R_{U}$ is a Schatten $p$-class operator if and only if $\int_{\Omega} B_{U, \Omega}^{(2 p+1)}(z, z) d V(z)<\infty$. Furthermore,

$$
\left\|R_{U}\right\|_{S_{p}}=\left\|T_{U}\right\|_{S_{2 p}}^{1 / 2}=\left(\int_{\Omega} B_{U, \Omega}^{(2 p+1)}(z, z) d V(z)\right)^{1 / 4 p}
$$


Proof of Proposition 11. Since $T_{U}$ is a positive operator $T_{U}^{p}$ is a positive positive operator on $A^{2}(\Omega)$ for every positive real number $p$. Then for $z \in \Omega$ and $p>0$ we have

$$
0 \leq\left\langle T_{U}^{p} B_{\Omega}(., z), B_{\Omega}(., z)\right\rangle=T_{U}^{p} B_{\Omega}(., z)(z)=B_{U, \Omega}^{(p+1)}(z, z) .
$$

It is known (cf. the proof in [Zhu07, Theorem 6.4]) that the Schatten 1-norm of a positive operator $T$ on $A^{2}(\Omega)$ is given by:

$$
\|T\|_{S_{1}}=\int_{\Omega}\left\langle T B_{\Omega}(., z), B_{\Omega}(., z)\right\rangle d V(z),
$$

where the integrand is equal to the Berezin transform of the operator $T$ times $B_{\Omega}(z, z)$. Note that both sides of the above equation may be infinite. Using [Zhu07, Lemma 1.25] to compute $\left\|R_{U}\right\|_{S_{p}}$ in terms of $\left\|T_{U}^{2 p}\right\|_{S_{1}}$, we have

$$
\begin{aligned}
\left\|R_{U}\right\|_{S_{p}}^{4 p}=\left\|T_{U}\right\|_{S_{2 p}}^{2 p} & =\left\|T_{U}^{2 p}\right\|_{S_{1}} \\
& =\int_{\Omega}\left\langle T_{U}^{2 p} B_{\Omega}(., z), B_{\Omega}(., z)\right\rangle d V(z), \\
& =\int_{\Omega} B_{U, \Omega}^{(2 p+1)}(z, z) d V(z),
\end{aligned}
$$

where the last line follows from (34).

We end the paper by justifying the trace formula (44).

$$
\begin{aligned}
\left\|T_{U}\right\|_{S_{1}}=\int_{\Omega} B_{U, \Omega}^{(2)}(z, z) d V(z) & =\int_{\Omega} \int_{\Omega} \chi_{U}(\xi) B_{\Omega}(\xi, z) B_{\Omega}(z, \xi) d V(\xi) d V(z) \\
& =\int_{\Omega} \int_{U}\left|B_{\Omega}(z, \xi)\right|^{2} d V(\xi) d V(z) \\
\text { (Using Tonelli's theorem) } & =\int_{U} \int_{\Omega}\left|B_{\Omega}(\xi, z)\right|^{2} d V(\xi) d V(z) \\
& =\int_{U} B_{\Omega}(\xi, \xi) d V(\xi) .
\end{aligned}
$$

\section{REFERENCES}

[AAD01] Lev Aizenberg, Aydin Aytuna, and Plamen Djakov, Generalization of a theorem of Bohr for bases in spaces of holomorphic functions of several complex variables, J. Math. Anal. Appl. 258 (2001), no. 2, 429-447.

[And00] Mats Erik Andersson, An inverse problem connected to double orthogonality in Bergman spaces, Math. Proc. Cambridge Philos. Soc. 128 (2000), no. 3, 535-538.

[Ber70] Stefan Bergman, The kernel function and conformal mapping, revised ed., American Mathematical Society, Providence, R.I., 1970, Mathematical Surveys, No. V.

[Cat80] David Catlin, Boundary behavior of holomorphic functions on pseudoconvex domains, J. Differential Geom. 15 (1980), no. 4, 605-625 (1981).

[Cox98] H. S. M. Coxeter, Non-Euclidean geometry, sixth ed., MAA Spectrum, Mathematical Association of America, Washington, DC, 1998.

[CS01] So-Chin Chen and Mei-Chi Shaw, Partial differential equations in several complex variables, AMS/IP Studies in Advanced Mathematics, vol. 19, American Mathematical Society, Providence, RI, 2001.

[ČŞ18] Željko Čučković and Sönmez Şahutoğlu, Essential norm estimates for the $\bar{\partial}-n e u m a n n$ operator on convex domains and worm domains, Indiana Univ. Math. J. 67 (2018), no. 1, 267-292.

[Dal18] Gian Maria Dall'Ara, On noncompactness of the $\bar{\partial}-$ Neumann problem on pseudoconvex domains in $\mathbb{C}^{3}, \mathrm{~J} . \mathrm{Math}$. Anal. Appl. 457 (2018), no. 1, 233-247.

[Dav95] E. B. Davies, Spectral theory and differential operators, Cambridge Studies in Advanced Mathematics, vol. 42, Cambridge University Press, Cambridge, 1995.

[FS98] Siqi Fu and Emil J. Straube, Compactness of the $\bar{\partial}-$ Neumann problem on convex domains, J. Funct. Anal. 159 (1998), no. 2, 629-641. 
[GKK11] Robert E. Greene, Kang-Tae Kim, and Steven G. Krantz, The geometry of complex domains, Progress in Mathematics, vol. 291, Birkhäuser Boston, Inc., Boston, MA, 2011.

[GPS03] Björn Gustafsson, Mihai Putinar, and Harold S. Shapiro, Restriction operators, balayage and doubly orthogonal systems of analytic functions, J. Funct. Anal. 199 (2003), no. 2, 332-378.

[Gun66] R. C. Gunning, Lectures on Riemann surfaces, Princeton Mathematical Notes, Princeton University Press, Princeton, N.J., 1966.

[JP93] Marek Jarnicki and Peter Pflug, Invariant distances and metrics in complex analysis, De Gruyter Expositions in Mathematics, vol. 9, Walter de Gruyter \& Co., Berlin, 1993.

[Kim03] Mijoung Kim, The $\bar{\partial}-$ Neumann operator and the Kobayashi metric, Ph.D. thesis, Texas A\&M University, 2003, available at http://hdl.handle.net/1969.1/94.

[Kim04] ㄴ. The $\bar{\partial}-$ Neumann operator and the Kobayashi metric, Illinois J. Math. 48 (2004), no. 2, 635-643.

[Lar86] James W. Lark, III, Spectral theorems for a class of Toeplitz operators on the Bergman space, Houston J. Math. 12 (1986), no. 3, 397-404.

[Lue81] Daniel H. Luecking, Inequalities on Bergman spaces, Illinois J. Math. 25 (1981), no. 1, 1-11.

[Lue84] Daniel Luecking, Closed ranged restriction operators on weighted Bergman spaces, Pacific J. Math. 110 (1984), no. 1, 145-160.

[MH71] B. S. Mitjagin and G. M. Henkin, Linear problems of complex analysis, Uspehi Mat. Nauk 26 (1971), no. 4 (160), 93-152.

[Nar95] Raghavan Narasimhan, Several complex variables, Chicago Lectures in Mathematics, University of Chicago Press, Chicago, IL, 1995, Reprint of the 1971 original.

[Pfl5] Peter Pflug, Quadratintegrable holomorphe Funktionen und die Serre-Vermutung, Math. Ann. 216 (1975), no. 3, 285-288.

[PP06] Gabriela Putinar and Mihai Putinar, Remarks on restriction eigenfunctions in $\mathbf{C}^{n}$, Electron. Trans. Numer. Anal. 25 (2006), 393-408.

[RSN90] Frigyes Riesz and Béla Sz.-Nagy, Functional analysis, Dover Books on Advanced Mathematics, Dover Publications, Inc., New York, 1990, Translated from the second French edition by Leo F. Boron, Reprint of the 1955 original.

[Sha79] H. S. Shapiro, Stefan Bergman's theory of doubly-orthogonal functions. An operator-theoretic approach, Proc. Roy. Irish Acad. Sect. A 79 (1979), no. 6, 49-58.

[ŞS06] Sönmez Şahutoğlu and Emil J. Straube, Analytic discs, plurisubharmonic hulls, and non-compactness of the $\bar{\partial}$ Neumann operator, Math. Ann. 334 (2006), no. 4, 809-820.

[Str10] Emil J. Straube, Lectures on th $l^{2}$-Sobolev theory of the $\bar{\partial}$-Neumann problem, ESI Lectures in Mathematics and Physics, European Mathematical Society (EMS), Zürich, 2010.

[Vas08] Nikolai L. Vasilevski, Commutative algebras of Toeplitz operators on the Bergman space, Operator Theory: Advances and Applications, vol. 185, Birkhäuser Verlag, Basel, 2008.

[Zhu07] Kehe Zhu, Operator theory in function spaces, second ed., Mathematical Surveys and Monographs, vol. 138, American Mathematical Society, Providence, RI, 2007.

(Debraj Chakrabarti) Department of Mathematics, Central Michigan University, Mt. Pleasant, Mi 48859, USA

E-mail address: chakr2d@cmich.edu USA

(Sönmez Şahutoğlu) Department of Mathematics and Statistics, University of Toledo, Toledo, OH 43606,

E-mail address: sonmez.sahutoglu@utoledo.edu 